\title{
Resonances en limite semiclassique et exposants de Lyapunov
}

\author{
C. Gérard et J. Sjöstrand \\ Département de Mathématiques, Université de Paris Sud, F-91405 Orsay, France
}

\begin{abstract}
We determine the width of resonance-free domains in the complex plane for the semiclassical Schrödinger operator $-h^{2} \Delta+V(x)$ when $h \rightarrow 0$, in terms of Lyapunov exponents for the associated classical flow.
\end{abstract}

\section{Introduction}

Soit $P=P(x, D, h), D=i^{-1} h \partial_{x}$, un opérateur différentiel sur $\mathbb{R}^{n}$ à coefficients analytiques qui vérifie les hypothèses générales de [5, Sect. 8], permettant de définir les résonances dans un petit voisinage de zéro dans le demi-plan inférieur. Le but de ce travail est de donner sous des hypothèses convenables une minoration de $-\operatorname{Im} z$ pour toute résonance $z$ quand $h \rightarrow 0$, en terme des propriétés du flot classique dans un voisinage de l'ensemble des trajectoires captées. L'étude des résonances crées par un point fixe (voir [9] et Briet et al. [11]) et par une trajectoire fermée hyperbolique (voir [5]) suggère que l'existence d'une bande sans résonances quand $h \rightarrow 0$ est liée à des propriétés d'hyperbolicité du flot classique. Dans ce travail nous étudions le cas plus général où l'ensemble des trajectoires captées pour le flot classique est contenu dans une varieté invariante, ayant une structure hyperbolique.

Soit $p(x, \xi)$ le symbole principal de $P$ en tant qu'opérateur $h$-pseudodifférentiel. Un cas particulièrement intéressant est celui où $P=-h^{2} \Delta+V(x), p=\xi^{2}+V(x)$. Dans l'appendice de [4], nous avons défini pour $\varepsilon_{0}$ assez petit, l'ensemble des trajectoires captées $K=\left\{\varrho \in p^{-1}\left(\left[-\varepsilon_{0}, \varepsilon_{0}\right]\right)\right.$; $\exp \left(t H_{p}\right)(\varrho)$ ne tend pas vers l'infini quand $t \rightarrow+\infty$ ou quand $t \rightarrow-\infty\}$. Ici $H_{p}$ désigne le champ hamiltonien associé à $p$. Pour $|t| \leqq \varepsilon_{0}$ on pose aussi $K_{t}=K \cap p^{-1}(t)$. Nous allons supposer que $K_{0}$ est contenu dans une variété symplectique localement $H_{p}$-invariante par rapport à laquelle le flot a une structure hyperbolique:

(H) Dans un voisinage ouvert $\Omega$ de $K$ dans $T^{*}\left(\mathbb{R}^{n}\right)$, il existe une sous-variété fermée symplectique de classe $C^{1}, \Sigma$, qui contient $K_{0}$ et telle que $H_{p}$ est tangent à $\Sigma$ en tout point. De plus on suppose qu'il existe deux sous-fibrés vectoriels $N_{+}$et $N_{-}$ de $T_{\Sigma}\left(T^{*} \mathbb{R}^{n}\right)$ de classe $C^{0}$ tels que:

$\left(\mathrm{H}_{1}\right) T_{\varrho} \Sigma^{\sigma}=N_{+, \varrho} \oplus N_{-, \varrho}$ en tout point $\varrho$ de $\Sigma$. 
Ici $T_{Q} \Sigma^{\sigma}$ désigne l'orthogonal de $T_{0} \Sigma$ pour la forme symplectique. $t \in \mathbb{R}$.

$\left.\left(\mathrm{H}_{2}\right) N_{+}\right|_{K_{0}}$ et $\left.N_{-}\right|_{K_{0}}$ sont invariants par les applications $d\left(\exp \left(t H_{p}\right)\right)$, pour

$\left(\mathrm{H}_{3}\right)$ Si on choisit des normes sur $N_{+, \varrho}$ et $N_{-, \varrho}$ dépendant continument de $\varrho$, il existe $C_{0}>1$ et $T_{0}>0$ tels que: $\left\|d\left(\exp \left( \pm\left(-T_{0}\right) H_{p}\right)\right)\left(v_{ \pm}\right)\right\| \leqq C_{0}^{-1}\left\|v_{ \pm}\right\|, \forall v_{ \pm} \in N_{ \pm . Q}$, $\varrho \in K_{0}$.

Le flot $d\left(\exp \left(t H_{p}\right)\right)$ est donc exponentiellement expansif sur $\left.N_{+}\right|_{K_{0}}$ et exponentiellement contractif sur $\left.N_{-}\right|_{K_{0}}$, pour $t \rightarrow+\infty$. Comme $d\left(\exp \left(t H_{p}\right)\right)$ conserve la forme symplectique, on en déduit que $N_{+, \varrho}$ et $N_{-. \varrho}$ sont isotropes et de même dimension, et donc des sous-espaces lagrangiens de $T_{\varrho} \Sigma^{\sigma}$, d'après (H1), en dualité pour la forme symplectique. $\operatorname{Sidim} \Sigma=2 n-2 d$, fixons une $d$-densité de volume $\neq 0$ sur $N_{+, \varrho}$ dépendant continument de $\varrho$, et prenons sur $N_{-, \varrho}$ le volume dual tel que le produit des deux volumes donne le volume symplectique sur $T_{\varrho} \Sigma^{\sigma}$. Pour $\varrho \in K_{0}$, on peut alors définir:

Alors on a:

$$
D_{ \pm}(\varrho, t)=\left.\operatorname{det}\left(d\left(\exp \left(t H_{p}\right)\right)\right)\right|_{N_{ \pm, \varrho}} .
$$

$$
D_{+}(\varrho, t) D_{-}(\varrho, t)=1 .
$$

D'après (H3), il existe $d>0, C>0$, tels que:

$$
D_{+}(\varrho, t) \geqq C^{-1} \exp (d t), \text { pour } \varrho \in K_{0}, t \geqq 0 .
$$

Soit $d_{0} \in \mathbb{R}_{+}$le sup de tous les $d>0$ vérifiant (0.3) pour un $C=C(d)>0$. On remarque que $d_{0}$ ne dépend pas du choix de densité sur les fibres de $N_{+}$. En fait $d_{0}$ est égal à $\inf _{\varrho \in K_{0}}(-\lambda(\varrho))$, où $\lambda(\varrho)$ est l'exposant de Lyapunov $d$-dimensionel quand $t \rightarrow+\infty$ associé au flot sur $\left.N_{-}\right|_{K_{0}}$ défini par $\left.d\left(\exp \left(t H_{p}\right)\right)\right|_{N_{-}}$(voir [7]).

Le résultat principal de ce travail est alors le théorème suivant:

Théorème 0.1. Pour tout $\delta>0$, il existe $\varepsilon>0$ tel que $P$ n'a pas de résonances dans $[-\varepsilon,+\varepsilon]-i\left[0,(1-\delta) d_{0} h / 2\right]$ pour $0<h<\varepsilon$.

Donnons maintenant quelques exemples. On vérifiera facilement que le Théorème 0.1 donne le résultat optimal dans la situation considérée dans [4] et $[9,11]$.

Exemple 1. Comme dans [9] on suppose que $K_{0}$ est réduit à un point fixe $\varrho_{0}$ de $H_{p}$, tel que la matrice fondamentale $G_{p}$, (qui est le linéarisé de $H_{p}$ ) en $\varrho_{0}$ a au moins une valeur propre de partie réelle $\neq 0$. Soit alors $\Sigma$ une variété centre, passant par $\varrho_{0}$, $H_{p}$-invariante (localement), et tangente en $\varrho_{0}$ à la somme des espaces propres de $G_{p}$ associés aux valeurs propres imaginaires pures ou nulles (voir [2, appendix C]). On choisit les fibrés $N_{+}$et $N_{-}$au dessus de $\Sigma$, tels que la fibre en $\varrho_{0}$ de $N_{+}\left(N_{-}\right)$soit la somme des espaces propres associés aux valeurs propres de partie réelle positive (négative). La constante $d_{0}$ est alors égale à $\operatorname{Re}\left(\lambda_{1}+\ldots+\lambda_{d}\right)$, où $\lambda_{1}, \ldots, \lambda_{d}$ sont les valeurs propres de $G_{p}$ à partie réelle positive.

Exemple 2. On suppose que $K_{0}$ est une trajectoire fermée $\gamma_{0}$ de période minimale $T_{0}>0$, telle qu'il existe au moins une paire de valeurs propres de l'application de Poincaré linéarisée $D_{p}$ de module différent de 1 . On prend pour $\Sigma$ une variété centre de $\gamma_{0}$ (voir [2, appendix C]). Dans le cas considéré dans [4] où $\gamma_{0}$ est 
hyperbolique, $\Sigma$ est simplement la réunion de la famille à un paramètre de trajectoires fermées $\gamma_{\varepsilon} \subset p^{-1}(\varepsilon)$ qui passe par $\gamma_{0}$. On prend $N_{+}$et $N_{-}$tels que les fibres au dessus des points de $\gamma_{0}$ sont les espaces naturellement associés aux sommes des espaces propres qui correspondent respectivement aux valeurs propres de module $>1$ et de module $<1$ de $D_{p}$. La constante $d_{0}$ est alors égale à $T_{0}^{-1} \log \left(\left|\theta_{1}\right| \ldots\left|\theta_{d}\right|\right)$, où $\theta_{1}, \ldots, \theta_{d}$ désigne les valeurs propres de $D_{p}$ de module $>1$. Le résultat de [4] montre que le Théorème 0.1 est optimal dans la situation de [4].

Exemple 3. On suppose que $K \cap p^{-1}\left(\left[-\varepsilon_{0}, \varepsilon_{0}\right]\right)=\Sigma \cap p^{-1}\left(\left[-\varepsilon_{0}, \varepsilon_{0}\right]\right)$, où $\Sigma$ est une variété $C^{1} r$-normalement hyperbolique et symplectique. Cette situation est discutée en détail dans l'appendice A.1.

Exemple 4. On donne maintenant un exemple où l'ensemble $K$ n'est pas une variété. Il est clair qu'on peut construire sur le même modèle beaucoup d'autres exemples avec des ensembles $K$ pathologiques.

On prend $p(x, \xi)=\xi^{2}+V(x)$ avec $x=\left(x_{1}, x_{2}, x^{\prime \prime}\right)$,

$$
V(x)=x_{1}^{3}-3 x_{1} x_{2}^{2}-\sum_{1 \leqq j \leqq n-2} m_{j}(x) x_{j}^{\prime \prime 2}, \quad \text { et } \quad m_{j}(x)>0 .
$$

Alors il est facile de voir qu'on peut choisir les $m_{j}$ à l'infini pour que $K_{0}$ soit inclus dans la variété symplectique $\Sigma=\left\{x^{\prime \prime}=\xi^{\prime \prime}=0\right\}$.

Le flot de $H_{p}$ sur $\Sigma$ est donné par le hamiltonien $\xi_{1}^{2}+\xi_{2}^{2}+x_{1}^{3}-3 x_{1} x_{2}^{2}$. Il est alors bien connu que l'ensemble des trajectoires captées pour le potentiel $x_{1}^{3}-3 x_{1} x_{2}^{2}$ (selle de singe) n'est pas une variété et contient par exemple une infinité d'orbites périodiques.

Pour démontrer le théorème, on utilise une méthode dépendant du temps, en écrivant la résolvante $(P-z)^{-1}$ comme $i h^{-1} \int_{0}^{\infty} e^{-i t(P-z) / h} d t$, pour $\operatorname{Im} z>0$. On montre essentiellement que $e^{-i t P / h}$ décroit exponentiellement quand $t \rightarrow \infty$, comme opérateur dans les espaces introduits par Helffer et Sjöstrand [5], et ceci entraine que la résolvante existe encore pour $z$ dans une bande dans le demiplan inférieur. Bien entendu $P$ n'est pas autoadjoint comme opérateur dans les espaces de [5], mais peut être considéré comme un opérateur pseudo-différentiel dont le symbole principal $p$ vérifie (avec un choix convenable de fonction fuite): $-\operatorname{Im}(p)(\varrho) \sim \operatorname{dist}(\varrho, \Sigma)^{2}$. Dans une telle situation, les specialistes des équations aux dérivées partielles, ont tendance à utiliser des inégalités du type de Gårding ou de Melin, pour minorer l'opérateur - $\operatorname{Im}(P)$. Dans notre cas, l'approche qui consiste à majorer la norme de l'opérateur intégral de Fourier, $e^{-i t P / h}$ quand $t \rightarrow \infty$, s'est revelée plus efficace, et moins dépendant d'un choix d'espace raffiné. (De plus, les constantes obtenues par cette méthode se relient directement à des invariants du flot classique du type exposants de Lyapunov.) Techniquement, la démonstration est basée sur les travaux $[5,8]$, qui fournissent aussi un cadre pour des arguments plus classiques du type localisation dans des boules convenables dans l'espace de phase. On peut dire que l'hypothèse $(\mathrm{H})$, qui prescrit une certaine diffusion pour les trajectoires classiques, empdeche la norme $L^{2}$ de $e^{-i t P / h} u$ rester concentrée près de $K_{0}$ pendant longtemps, et la force à suivre les trajectoires sortantes. Comme les espaces de [5] ont des normes très faibles près de ces trajectoires, on obtient une 
décroissance exponentielle. Plus concrètement, $d_{0}$ apparait comme un taux de décroissance exponentielle pour une amplitude dans la description de $e^{-i t P / h}$ comme opérateur intégral de Fourier.

Le plan de l'article est le suivant: Dans la Sect. 1 on construit une fonction fuite locale $G$ telle que $H_{p} G \sim \operatorname{dist}(\cdot, \Sigma)^{2}$. Ceci permet de se restreindre à la construction de $e^{-i t P / h}$ dans un voisinage microlocal de $\Sigma$ d'épaisseur $C h^{\varepsilon}$, avec $1 / 3<\varepsilon<1 / 2$. Dans la Sect. 2 on estime la norme de certains opérateurs intégraux de Fourier dans le domaine complexe en fonction de la norme de leurs linéarisés. Dans la Sect. 3, on étudie des linéarisés de $e^{-i t P / h}$. Dans la Sect. 4 on démontre le théorème en établissant une inégalité à priori dans des espace à poids microlocaux à l'aide des Sects. 2 et 3. On a rassemblé dans un appendice certains résultats géométriques.

\section{Construction d'une fonction fuite locale}

On choisit des normes euclidiennes sur $N_{+, o}$ dépendant continument de $\varrho$. On peut alors trouver (voir l'appendice) une fonction $G_{0} \in C^{2}(\Omega)$ s'annulant à l'ordre 2 sur $\Sigma$, telle que le Hessien transversal de $G_{0}$ soit donné en tout point de $\Sigma$ par:

$$
\left(G_{0}\right)_{\varrho}^{\prime \prime}\left(v_{+}+v_{-}\right)=\left\|v_{+}\right\|^{2}-\left\|v_{-}\right\|^{2} .
$$

Si $\Phi_{t}=d\left(\exp \left(t H_{p}\right)\right)$, alors pour $T>0$ assez grand on a:

$$
\left(G_{0}^{\prime \prime} \circ \Phi_{t}-G_{0}^{\prime \prime}\right)_{\varrho}(v) \geqq C^{-1}\|v\|^{2}, v \in T_{\varrho} \Sigma^{\sigma}, \varrho \in K_{0} .
$$

Si on fixe $T>0$ et restreint $\Omega$ suffisament autour de $K_{0}$, on a encore (1.2) pour $\varrho \in \Sigma$. Restreignant encore $\Omega$ on obtient:

$$
G_{0} \circ \varphi_{T}-G_{0} \sim \operatorname{dist}(\cdot, \Sigma)^{2} \text { dans } \Omega .
$$

Ici $\varphi_{t}=\exp \left(t H_{p}\right)$. Soit maintenant

$$
G=\int_{0}^{T} G_{0} \circ \varphi_{s} d s .
$$

Alors par un calcul simple, on a: $H_{p} G=G_{0} \circ \varphi_{T}-G_{0}$. On a donc trouvé $G \in C^{2}(\Omega)$, $\Omega$ voisinage de $K_{0}$, tel que:

$$
G=O(1) \operatorname{dist}(\cdot, \Sigma)^{2}, H_{p} G \sim \operatorname{dist}(\cdot, \Sigma)^{2} .
$$

Comme $K \rightarrow K_{0}$ quand $\varepsilon_{0} \rightarrow 0$, on montre facilement à partir de (1.5) que $K \subset \Sigma$, si $\varepsilon_{0}>0$ est assez petit. En effet, si $\varrho \in(\Omega \backslash \Sigma) \cap p^{-1}\left(\left[-\varepsilon_{0}, \varepsilon_{0}\right]\right)$ et si par exemple $G(\varrho) \geqq 0$, alors $t \rightarrow G\left(\exp \left(t H_{p}\right)(\varrho)\right)$ est strictement croissant sur tout intervalle [0,T[, tel que $\exp t H_{p}(\varrho) \in \Omega$ pour $0 \leqq t<T$. On en conclut que exp $t H_{p}(\varrho)$ finit par quitter $\Omega$ au bout d'un temps fini, et donc $\varrho \notin K$, comme $K$ est invariant pour $H_{p}$ et contenu dans $\Omega$.

Utilisant les arguments élémentaires de [4], appendice, on voit que $G$ peut être étendue en une fonction fuite globale (au sens de [5]), $C^{\infty}$ en dehors d'un petit voisinage de $\Sigma$ et vérifiant $H_{p} G>0$ dans $p^{-1}\left(\left[-\varepsilon_{0}, \varepsilon_{0}\right]\right) \backslash \Sigma$. 


\section{Norme des operateurs oscillants dans le domaine complexe}

Soit $\varphi(x, y, \theta)$ une phase non dégénérée définie près de $\left(x_{0}, y_{0}, \theta_{0}\right) \in \mathbb{C}^{n+n+N}$ telle que $d_{\theta} \varphi=0$ en $\left(x_{0}, y_{0}, \theta_{0}\right)$. Non dégénérée signifie que les différentielles $d \partial_{\theta_{1}} \varphi, \ldots, d \partial_{\theta_{N}} \varphi$ sont linéairement indépendantes. On suppose aussi que $\varphi$ engendre un germe de transformation canonique $H_{A}$ :

$$
\left(y, \partial_{y} \varphi(x, y, \theta)\right) \rightarrow\left(x, \partial_{x} \varphi(x, y, \theta)\right), \partial_{\theta} \varphi=0 .
$$

$\mathrm{Si}$

$$
a(x, y, \theta, h) \sim a_{0}(x, y, \theta)+a_{1}(x, y, \theta) h+\ldots
$$

est un symbole analytique classique d'ordre 0 , on peut considerer, comme dans [8], l'opérateur oscillant:

$$
A u(x, h)=h^{-(n+N) / 2} \iint e^{i \varphi(x, y \cdot \theta) / h} a(x, y, \theta, h) u(y) d y d \theta .
$$

Si on voit d'abord $A$ comme un objet formel, on lui associe son linéarisé formel $A\left(y_{0}, \eta_{0}\right)$ :

$$
A\left(y_{0}, \eta_{0}\right) u(x)=\iint e^{i \varphi^{\prime \prime}(x, y, \theta)} a_{0}\left(x_{0}, y_{0}, \theta_{0}\right) u(y) d y d \theta,
$$

où $\varphi^{\prime \prime}$ désigne le hessien de $\varphi$ en $\left(x_{0}, y_{0}, \theta_{0}\right)$. $\varphi^{\prime \prime}$ est une phase non dégénérée, définie globalement sur $\mathbb{C}^{2 n+N}$ et la transformation canonique associée est $d H_{A}\left(y_{0}, \eta_{0}\right): \mathbb{C}^{2 n} \rightarrow \mathbb{C}^{2 n}$. Formellement, on obtient $A\left(y_{0}, \eta_{0}\right)$ par un passage à la limite de la manière suivante: on pose $u(y)=e^{i\left(y-y_{0}\right) \eta_{0} / h} \tilde{u}(\tilde{y}), v(x)=e^{i\left(x-x_{0}\right) \xi_{0} / h} \tilde{v}(\tilde{x})$, avec $x=x_{0}+h^{1 / 2} \tilde{x}, y=y_{0}+h^{1 / 2} \tilde{y}, \theta=\theta_{0}+h^{1 / 2} \tilde{\theta}$. Si $A u=v$, alors on $a$ :

$$
\tilde{v}(\tilde{x})=\iint e^{i\left[-\left(x-x_{0}\right) \xi_{0}+\varphi(x, y, \theta)+\left(y-y_{0}\right) \eta_{0}\right] / h} a(x, y, \theta, h) \tilde{u}(\tilde{y}) d \tilde{y} d \tilde{\theta} .
$$

Ici la phase devient, $\varphi\left(x_{0}, y_{0}, \theta_{0}\right)+h \varphi^{\prime \prime}(\tilde{x}, \tilde{y}, \widetilde{\theta})+O\left(h^{3 / 2}\right)$, et on a donc:

$$
\tilde{v}(\tilde{x})=e^{i \varphi\left(x_{0}, y_{0}, \theta_{0}\right) / h} \iint e^{i\left(\varphi^{\prime \prime}(\tilde{x}, \tilde{y}, \tilde{\theta})+O\left(h^{1 / 2}\right)\right)}\left(a_{0}\left(x_{0}, y_{0}, \theta_{0}\right)+O\left(h^{1 / 2}\right)\right) \tilde{u}(\tilde{y}) d \tilde{y} d \tilde{\theta} .
$$

Formellement le menbre de droite converge vers:

$$
e^{i \varphi\left(x_{0}, y_{0}, \theta_{0}\right) / h} A\left(y_{0}, \eta_{0}\right) \tilde{u}(\tilde{x}) .
$$

Si $B$ est un deuxième opérateur oscillant:

$$
B v(z, h)=h^{-(n+M) / 2} \iint e^{i \psi(z, x, w) / h} b(z, x, w, h) v(x) d x d w,
$$

$H_{B}\left(x_{0}, \xi_{0}\right)=\left(z_{0}, \zeta_{0}\right)$, alors on peut écrire la composition formelle $B \circ A$ comme un opérateur oscillant de phase $\psi(z, x, w)+\varphi(x, y, \theta)$ et d'amplitude $b(z, x, w, h)$ $\times a(x, y, \theta, h)$, où on compte $(x, w, \theta)$ comme variables de fibre. Il est alors évident que:

$$
(B \circ A)\left(y_{0}, \eta_{0}\right)=B\left(x_{0}, \xi_{0}\right) \circ A\left(y_{0}, \eta_{0}\right),
$$

si on définit le composé des linéarisés de la même manière. Pour arriver à une discussion moins formelle, on se donne maintenant deux fonctions strictement plurisousharmoniques $\Phi_{1}$ et $\Phi_{2}$ de classe $C^{2}$, définies dans des voisinages de $y_{0}, x_{0}$, telles que $\left(x_{0}, \xi_{0}\right) \in \Lambda_{\Phi_{2}},\left(y_{0}, \eta_{0}\right) \in \Lambda_{\Phi_{1}}$, où $\Lambda_{\Phi_{j}}=\left\{\left(x,(2 / i) \partial_{x} \Phi_{j}\right)\right\}$. On suppose aussi que $H_{A}\left(\Lambda_{\Phi_{1}}\right)=\Lambda_{\Psi}$ où $\Psi$ est st.pl.sh. de classe $C^{2}$, avec:

$$
\Psi(x) \leqq \Phi_{2}(x), \Psi\left(x_{0}\right)=\Phi_{2}\left(x_{0}\right)=-\operatorname{Im} \varphi\left(x_{0}, y_{0}, \theta_{0}\right)+\Phi_{1}\left(y_{0}\right) .
$$


On sait alors ([8]) que:

$$
\Psi(x)=\text { v.c. }(y, \theta)\left(-\operatorname{Im} \varphi(x, y, \theta)+\Phi_{1}(y)\right),
$$

où v.c. désigne la valeur critique par rapport aux variables en indice, et le point critique $(y(x), \theta(x))$ correspondant est non dégénéré de signature 0 . Pour simplifier notre discussion, nous ferons aussi l'hypothèse suivante qui sera vérifiée dans les Sects. 3 et 4 :

il existe une sous variété $\Sigma_{1} C A_{\Phi_{1}}$ de classe $C^{1}$, passant par $\left(y_{0}, \eta_{0}\right)$, telle que si $\Sigma_{2}=H_{A}\left(\Sigma_{1}\right)$, on $a: \Phi_{2}-\Psi \sim \operatorname{dist}\left(\cdot, \Pi_{x} \Sigma_{2}\right)^{2}$.

Ici $\Pi_{x}$ designe la projection naturelle sur l'espace des $x$.

Soient $\Omega_{1}$ et $\Omega_{2}$ deux petits voisinages de $x_{0}, y_{0}$ tels que: $\Omega_{2} \subset \subset \Pi_{x} H_{A}\left(\Lambda_{\Phi_{1}}\right.$ $\left.\cap \Pi_{x}^{-1} \Omega_{1}\right)$. On pose $L_{\Phi}^{2}(\Omega)=\left\{u ; e^{-\Phi / h} u \in L^{2}(\Omega)\right\}, H_{\Phi}(\Omega)=L_{\Phi}^{2}(\Omega) \cap\{$ les fonctions holomorphes dans $\Omega\}$, munis de la norme naturelle. On peut alors définir $A$ comme opérateur de norme $O(1): H_{\Phi_{1}}\left(\Omega_{1}\right) \rightarrow L_{\Phi_{2}}^{2}\left(\Omega_{2}\right)$ en choisissant grâce à $(2.5)$ un bon contour dans (2.1) c.a.d. un cycle d'intégration $C^{\infty} \Gamma_{x}$, qui dépend de manière $C^{\infty} \mathrm{de}$ $x$ tel que:

$$
(y, \theta) \in \Gamma_{x} \Rightarrow-\Psi(x)-\operatorname{Im} \varphi(x, y, \theta)+\Phi_{1}(y) \sim-|y-y(x)|^{2}-|\theta-\theta(x)|^{2} .
$$

On s'intéresse alors à la norme de $A$ comme opérateur $H_{\Phi_{1}}\left(\Omega_{1}\right) \rightarrow L_{\Phi_{2}}^{2}\left(\Omega_{2}\right)$. De manière générale $A$ est modifié par un exponentiellement petit si $\Gamma_{x}$ est modifié et $\bar{\partial} A u$ est exponentiellement petit dans $L_{\Phi_{2}}^{2}\left(\Omega_{2}\right)$. Modifiant $A u$ par $O\left(h^{\infty}\right)$ dans $L_{\Phi_{2}}^{2}\left(\Omega_{2}\right)$ on peut restreindre (en utilisant $(2.7)$ ), le contour d'intégration $\Gamma_{x}$ à:

$$
|y-y(x)|+|\theta-\theta(x)| \leqq h^{b} \quad \text { si } \quad b<1 / 2 .
$$

Si $\Psi^{\prime \prime}, \Phi_{j}^{\prime \prime}$ désignent les Hessiens aux points $x_{0}, y_{0}$ il est maintenant clair que $A\left(x_{0}, \eta_{0}\right)$ est bien défini comme opérateur borné:

$$
H_{\Phi_{1}^{\prime \prime}}\left(\mathbb{C}^{n}\right) \rightarrow H_{\psi^{\prime \prime}}\left(\mathbb{C}^{n}\right) \subset H_{\Phi_{2}^{\prime \prime}}\left(\mathbb{C}^{n}\right) \text {, en prenant comme contour dans (2.2): }
$$

$(\tilde{y}(x), \widetilde{\theta}(x))+T_{\left(y_{0}, \theta_{0}\right)} \Gamma_{x_{0}}$, où $(\tilde{y}(x), \widetilde{\theta}(x))$ est le point critique de:

$$
(\tilde{y}, \tilde{\theta}) \rightarrow-\operatorname{Im} \varphi^{\prime \prime}(\tilde{x}, \tilde{y}, \tilde{\theta})+\Phi_{2}^{\prime \prime}(\tilde{y}) .
$$

On fixe $a \in] 1 / 3,1 / 2\left[\right.$. Soit $V_{2} \subset B\left(x_{0}, h^{a}\right)$ un voisinage de $x_{0}$ et posons $V_{1}=\Pi_{y} H_{A}^{-1}\left(\Pi_{x}^{-1} V_{2} \cap \Lambda_{\Psi}\right)$. On veut approcher $A u$ dans $V_{2}$ par une expression faisant intervenir le linéarisé $A\left(y_{0}, \eta_{0}\right)$. Pour simplifier nos formules au maximum, on supposera sans perte de généralité que $\xi_{0}=\eta_{0}=0, \Phi_{1}\left(y_{0}\right)=\Phi_{2}\left(x_{0}\right)=0$. On prend $b$ dans (2.8) tel que $a<b<1 / 2$.

$\mathrm{Si}$ on fait les mêmes substitutions qu'au début de cette section, on trouve:

$$
\tilde{v}(\tilde{x})=\iint_{\tilde{\Gamma}} e^{i\left(\varphi^{\prime \prime}\right.}\left(\tilde{x}^{\left., \tilde{y}, \tilde{\theta})+O\left(h^{3 a-1}\right)\right)} C a_{0}\left(x_{0}, y_{0}, \theta_{0}\right)+O\left(h^{a}\right)\right) \tilde{u}(\tilde{y}) d \tilde{y} d \tilde{\theta},
$$

où $\tilde{\Gamma}$ est l'image de $\Gamma_{x}$ par les substitutions. Nous avons alors:

$$
\begin{gathered}
\tilde{x} \in \tilde{V}_{2} \subset B\left(0, h^{a-1 / 2}\right) \text { et pour }(\tilde{y}, \tilde{\theta}) \in \tilde{\Gamma} \text {, on } a \\
|\tilde{y}-\tilde{y}(\tilde{x})|+|\tilde{\theta}-\tilde{\theta}(\tilde{x})| \leqq C h^{b-1 / 2} \\
-\psi^{\prime \prime}(\tilde{x})-\operatorname{Im} \varphi^{\prime \prime}(\tilde{x}, \tilde{y}, \tilde{\theta})+\Phi_{1}^{\prime \prime}(\tilde{y}) \sim-\left(|\tilde{y}-\tilde{y}(\tilde{x})|^{2}+|\tilde{\theta}-\tilde{\theta}(\tilde{x})|^{2}+O\left(h^{3 a-1}\right)\right) .
\end{gathered}
$$


Soient $\tilde{V}_{1}, \tilde{V}_{2}$ les voisinages de 0 dans $\mathbb{C}^{n}$ que l'on obtient par $x \rightarrow \tilde{x}, y \rightarrow \tilde{y}$. Pour alléger les notations, on écrit $(x, y, \theta)$ à la place de $(\tilde{x}, \tilde{y}, \widetilde{\theta})$ et $a_{0,0}$ à la place de $a_{0}\left(x_{0}, y_{0}, \theta_{0}\right) . \mathrm{Si}$

$$
v_{1}(x)=\int_{\tilde{\Gamma}} e^{i \varphi^{\prime \prime}(x, y, \theta)} a_{0,0} \tilde{u}(y) d y d \theta
$$

alors:

$$
\left\|\tilde{v}-v_{1}\right\|_{\Phi_{2}^{\prime}, \tilde{V}_{2}} \leqq C h^{3 a-1}\|\tilde{u}\|_{\Phi_{1}^{\prime}, \tilde{V}_{1}+B\left(0, C h^{b-1 / 2}\right)},
$$

où \|\|$_{\Phi_{i}, V}$ désigne la norme dans $H_{\Phi_{i}}(V)$.

Pour comparer (2.12) avec $A\left(y_{0}, \eta_{0}\right)$ on a besoin d'approcher $\tilde{u}$ par une fonction entière. Soit $\chi \in C^{\infty}\left(\widetilde{V}_{1}+B\left(0,2 C h^{b-1 / 2}\right)\right.$ égale à 1 près de $\widetilde{V}_{1}+B\left(0, C h^{b-1 / 2}\right)$, telle que $\partial^{\alpha} \chi=O\left(h^{|\alpha|(1 / 2-b)}\right)$, et telle que $0 \leqq \chi \leqq 1$. Alors $\bar{\partial} \chi \tilde{u}=\tilde{u} \bar{\partial} \chi=O\left(h^{1 / 2-b}\|\tilde{u}\|\right)$ dans $L_{\Phi_{1}^{\prime \prime}}^{2}$, et on peut trouver $w \in L_{\Phi_{1}^{\prime \prime}}^{2}\left(\mathbb{C}^{n}\right)$ avec norme

$$
O\left(h^{1 / 2-b}\|\tilde{u}\|_{\Phi_{1}^{\prime \prime}, \tilde{V}_{1}+B\left(0,2 C h^{b-1 / 2}\right)}\right),
$$

telle que $\bar{\partial} w=\bar{\partial} \chi \tilde{u}$. Alors $u_{1}=\chi \tilde{u}-w \in H_{\Phi_{1}^{\prime \prime}}$, et on $a$ :

$$
\begin{gathered}
\left\|u_{1}\right\|_{\Phi_{1}^{\prime \prime}} \leqq\left(1+O\left(h^{1 / 2-b}\right)\right)\|\tilde{u}\|_{\Phi_{1}^{\prime}, \tilde{V}_{1}+B\left(0,2 C h^{b-1 / 2)}\right.} . \\
\left\|\tilde{u}-u_{1}\right\|_{\Phi_{1}^{\prime}, \tilde{V}_{1}+B\left(0, C h^{b-1 / 2}\right)} \leqq C h^{1 / 2-b}\|\tilde{u}\|_{\Phi_{1}^{\prime \prime}, \tilde{V}_{1}+B\left(0,2 C h^{b-1 / 2}\right)} .
\end{gathered}
$$

Si $v_{2}=\iint_{\tilde{\Gamma}} e^{i \varphi^{\prime \prime}} a_{0,0} u_{1} d y d \theta, v_{3}=A\left(y_{0}, \eta_{0}\right) u_{1}$, on $a$ :

$$
\left\|v_{1}-v_{2}\right\|_{\Phi_{2}^{\prime}, \hat{V}_{2}} \leqq C h^{1 / 2-b}\|\tilde{u}\|_{\Phi_{1}^{\prime \prime} . \tilde{V}_{1}+B\left(0,2 C h^{b-1 / 2}\right)},
$$

et de même pour $v_{2}-v_{3}$.

Avec (2.13), on obtient donc:

$$
\left\|\tilde{v}-A\left(y_{0}, \eta_{0}\right) u_{1}\right\|_{\Phi_{2}^{\prime}, \tilde{V}_{2}} \leqq C h^{c}\|\tilde{u}\|_{\Phi_{1}^{\prime}, \hat{V}_{1}+B\left(0,2 C h^{b-1 / 2}\right)},
$$

où $c=\min (1 / 2-b, 3 a-1)>0$. (2.14) donne alors:

$$
\|\tilde{v}\|_{\Phi_{2}^{\prime}, \check{V}_{2}} \leqq\left(\left\|A\left(y_{0}, \eta_{0}\right)\right\|+C h^{c}\right)\|\tilde{u}\|_{\Phi_{1}^{\prime} . \check{V}_{1}+B\left(0,2 C h^{b-1 / 2}\right)},
$$

où $\left\|A\left(y_{0}, \eta_{0}\right)\right\|$ désigne la norme dans $L\left(H_{\Phi_{1}^{\prime}}, H_{\Phi_{2}^{\prime}}\right)$. Revenant dans les coordonnées de départ et utilisant que $\Phi_{j}-\Phi_{j}^{\prime \prime}=O\left(h^{3 a}\right)$ dans $V_{j}$, on trouve:

$$
\|A u\|_{\Phi_{2}, V_{2}} \leqq\left(\left\|A\left(y_{0}, \eta_{0}\right)\right\|+C h^{c}\right)\|u\|_{\Phi_{1} . V_{1}+B\left(0 . C h^{b-1 / 2}\right)} .
$$

Soient $x=\left(x^{\prime}, x^{\prime \prime}\right)$ des coordonnées locales réelles de classe $C^{1}$, centrées en $x_{0}$, telles que $\Pi_{x} \Sigma_{2}$ devienne $x^{\prime \prime}=0$. On suppose pour se fixer les idées que $\Omega_{2, a}=\Omega_{2}$ $\cap\left\{x ;\left|x^{\prime \prime}\right|<h^{a}\right\}=\Omega_{2}^{\prime} \otimes\left\{x^{\prime \prime} ;\left|x^{\prime \prime}\right|<h^{a}\right\}$. Pour $\alpha \in \Pi_{x} \Sigma_{2}$, on pose:

$$
V_{2, \alpha}=\left\{x ;\left|x^{\prime}-\alpha\right|<h^{a},\left|x^{\prime \prime}\right|<h^{a}\right\} .
$$

Si $d m(\alpha)=C h^{-2(n-d) a} d \alpha$, où $C>0$ est une constante convenable et $d \alpha$ désigne la mesure de Lebesgue dans les coordonnées $\alpha \in \mathbb{R}^{2(n-d)}$, alors:

$$
\int_{\Omega_{2}^{\prime}+B^{\prime}\left(0, h^{a}\right)} \mathbb{1}_{V_{2, \alpha}} d m(\alpha)=1 \text { dans } \Omega_{2, a} .
$$

Si $V_{1, \alpha}=\Pi_{x} H_{A}^{-1}\left(\Lambda_{\psi} \cap \Pi_{x}^{-1}\left(V_{2, \alpha}\right)\right)$, alors:

$$
\int_{\alpha \in \Omega_{2}^{\prime}+B^{\prime}\left(0, h^{a}\right)} \mathbb{1}_{V_{1 \times}+B\left(0, C h^{b}\right)} d m(x) \leqq 1+C . s t \cdot h^{b-a},
$$


et l'intégrale à gauche est une fonction à support dans $\Omega_{1}+B\left(0, C s t \cdot h^{a}\right)$. Dans l'estimation (2.18), on peut remplacer $V_{1}, V_{2}, A\left(y_{0}, \eta_{0}\right)$ par $V_{1, \alpha}, V_{2, \alpha}, A\left(y_{\alpha}, \eta_{\alpha}\right)$, où $\left(y_{\alpha}, \eta_{\alpha}\right)$ est le point avec $\Pi_{x} H_{A}\left(y_{\alpha}, \eta_{\alpha}\right)=\alpha$. Elevant cette estimation au carré pour tout $\alpha$, et intégrant par rapport à $\alpha$ sur $\Omega_{2}^{\prime}+B\left(0, h^{a}\right)$, on trouve à l'aide de (2.19), (2.20):

$$
\|A u\|_{\Phi_{2}, \Omega_{2, a}}^{2} \leqq\left(\sup _{(y, \eta) \in \Sigma_{1} \cap \Omega_{1}+B\left(0, C h^{a}\right)}\|A(y, \eta)\|+C h^{c}\right)\left(1+C h^{b-a}\right)\|u\|_{\Phi_{1}, \Omega_{1}+B\left(0, C h^{a}\right)}^{2} .
$$

Grâce à (2.6), (2.7), on obtient facilement:

$$
\|A u\|_{\Phi_{2}, \Omega_{2} \backslash \Omega_{2, a}}^{2} \leqq C e^{-h^{-d}}\|u\|_{\Phi_{1}, \Omega_{1}+B\left(0 . C h^{a}\right)}^{2},
$$

pour un $d>0$. On obtient:

Proposition 2.1. Pour tout $u \in H_{\Phi_{1}}(\Omega)$, on $a$ :

$$
\left.\|A u\|_{\Phi_{2}, \Omega_{2}} \leqq\left(\sup _{(y, \eta) \in \Sigma_{1} \cap \Pi_{x}^{-1}\left(\Omega_{1}+B\left(0, C h^{a}\right)\right.}\right)\|A(y, \eta)\|+C^{h c}\right)\|u\|_{\Phi_{1}, \Omega_{1}+B\left(0, C h^{a}\right)},
$$

pour $C>0, c>0$ indépendants de $u$ et de $h$.

Comme on le voit facilement à posteriori, cette proposition est valable aussi sans l'hypothèse sur $\Omega_{2, a}$ énoncée entre (2.18) et (2.19).

\section{Normes des linéarisés de $\exp (-i t P / h)$}

Soit $\widetilde{G} \in S^{1,1}$, (espace défini dans [5]), et $m(\alpha)$ une fonction poids comme dans le même travail. Localement $S^{1,1}$ coincide avec $C^{\infty}\left(\mathbb{R}^{2 n} ; \mathbb{R}\right)$ et $m$ est une fonction $C^{\infty}$ réelle strictement positive. Ce sont les seuls aspects qui joueront un rôle par la suite. Pour $\varepsilon$ assez petit, on a défini dans [5] un espace $H\left(\Lambda_{\varepsilon \tilde{G}}, m\right)$, muni de la norme:

$$
\|u\|_{\varepsilon \widetilde{G}, m}^{2}=\int_{\Lambda}|T u(\alpha, h)|^{2} m(\alpha)^{2} e^{-2 H(\alpha) / h} d \alpha,
$$

où $\Lambda=\Lambda_{\tilde{G}} \subset \mathbb{C}^{2 n}$ est donnée par:

$$
\operatorname{Im}(x, \xi)=\varepsilon H_{\check{G}}(\operatorname{Re}(x, \xi)),
$$

et $T$ est une certaine transformation de type Fourier-Bros-Iagolnitzer dont on n'a pas besoin de rappeler la définition ici. De même pour la fonction $H=\varepsilon_{\varepsilon} H_{\varepsilon}$.

Soit d'autre part:

$$
U u(x, h)=C_{0} h^{-n / 2} \int e^{-(x-y)^{2} / 2 h+x^{2} / 4 h} u(y) d y, \quad x \in \mathbb{C}^{n},
$$

une transformation de Fourier-Bros-Iagolnitzer standard. Considérée globalement $U$ est unitaire de $L^{2}\left(R^{n}\right)$ dans $H_{\Phi_{0}}\left(\mathbb{C}^{n}\right)$, où:

$$
H_{\Phi_{0}}\left(\mathbb{C}^{n}\right)=\left\{u: \mathbb{C}^{n} \rightarrow \mathbb{C} ; u \text { holomorphe, } \int|u|^{2} e^{-2 \Phi_{0} / h} L(d x)<\infty\right\} .
$$

Ici $\Phi_{0}=|x|^{2} / 4=\sup _{y \in R^{n}}-\operatorname{Im} \varphi(x, y)$, où $\varphi(x, y)=i\left((x-y)^{2}-x^{2} / 4\right)$. On a aussi $\Lambda_{\Phi_{0}}=H_{U}\left(\mathbb{R}^{2 n}\right)$, où $H_{U}$ est la transformation canonique complexe linéaire donnée par: $\left(y,-\partial_{y} \varphi\right) \rightarrow\left(x, \partial_{x} \varphi\right)$. 
Dans la suite on identifiera souvent un ensemble $A \subset \mathbb{R}^{2 n}$ avec $H_{U}(A) \subset A_{\Phi_{0}}$ où avec la projection: $\Pi_{x} H_{U}(A) \subset \mathbb{C}_{x}^{n}$ de cet ensemble. Alors pour $\varepsilon$ assez petit, on a au dessus de $\Omega$ :

$$
\begin{gathered}
H_{U}\left(\Lambda_{\varepsilon \tilde{G}}\right)=\Lambda_{\tilde{\Phi}_{\varepsilon}}, \quad \text { où } \quad \tilde{\Phi}_{\varepsilon} \in C^{\infty}(\Omega) \text { et: } \\
\tilde{\Phi}_{\varepsilon}-\Phi_{0}=\varepsilon \widetilde{G}+O\left(\varepsilon^{2}|\nabla \tilde{G}|^{2}\right) .
\end{gathered}
$$

(Ici on identifie $\widetilde{G}$ avec $\left.\widetilde{G} \circ\left(\left.\Pi_{x} \circ H_{U}\right|_{R^{2 n}}\right)^{-1}\right)$.

Il résulte de [5, Sect. 9], que pour $u \in H\left(\Lambda_{\varepsilon \tilde{G}}, m\right)$, on $a$ :

$$
\|U u\|_{\tilde{\Phi}_{\varepsilon}, \Omega} \leqq C\|u\|_{\varepsilon \tilde{G}, m} .
$$

Soit $\Omega_{2} \subset \subset \Omega$ un deuxième voisinage de $K_{0}$, que l'on identifiera aussi avec $\left\{\alpha \in \Lambda_{\varepsilon \tilde{G}} ; \operatorname{Re} \alpha \in \Omega_{2}\right\}$. On montre alors à l'aide des techniques de $[5,8]$, que pour $0 \leqq \varepsilon<\varepsilon_{0}$ avec $\varepsilon_{0}$ assez petit, il existe $\delta_{0}>0$ tel que:

$$
\|u\|_{\varepsilon \tilde{G}, \Omega_{2} . m} \leqq C\left(\|U u\|_{\tilde{\Phi}_{\varepsilon}, \Omega}+e^{-\delta_{0} / h}\|u\|_{\varepsilon \tilde{G}, C \Omega_{2} . m}\right),
$$

où de manière générale, la seminorme $\|u\|_{\varepsilon \tilde{G}, W, m}$ est définie comme dans (3.1) en intégrant seulement sur $W \subset \Lambda_{\varepsilon \tilde{G}}$. Une norme uniformément équivalente, quand $h \rightarrow 0$ pour $H\left(\Lambda_{\varepsilon \tilde{G}}, m\right)$ est donc donnée par:

$$
\|\boldsymbol{u}\|_{\varepsilon \tilde{\boldsymbol{\sigma}}, C \Omega_{2}, m}+\|U \mathcal{U}\|_{\tilde{\Phi}_{\varepsilon}, \Omega} .
$$

Si $G \in C^{2}\left(\mathbb{R}^{2 n} ; \mathbb{R}\right)$ est égale à $\widetilde{G}$ dans $C \Omega_{2}$ alors pour $\varepsilon>0$ assez petit $H_{U}\left(\Lambda_{\varepsilon G}\right)=\Lambda_{\Phi_{\varepsilon}}$, avec $\Phi_{\varepsilon} \in C^{2}, \Phi_{\varepsilon}=\widetilde{\Phi}_{\varepsilon}$ dans $\Omega \backslash \Omega_{2}$, et on $a$ :

$$
\Phi_{\varepsilon}-\Phi_{0}=\varepsilon G+O\left(\left.\varepsilon^{2} \nabla G\right|^{2}\right) .
$$

On pose pour $u \in H\left(\Lambda_{\varepsilon \tilde{G},}, m\right)$ :

$$
\|u\|_{\varepsilon G, m}=\|u\|_{\varepsilon \tilde{G}, C \Omega_{2}, m}+\|U u\|_{\Phi_{\varepsilon}, \Omega} .
$$

Cette norme est équivalente à la norme précédente mais pas uniformément quand $h \rightarrow 0$. On écrira $H(\varepsilon G, m)=H(\varepsilon \widetilde{G}, m)$. Dans la suite $G$ sera la fonction construite dans la Sect. 1 et $\widetilde{G}$ sera une fonction auxiliaire qu'on oubliera. Après conjuguaison par $U$, nous pouvons considerer $P$ comme un opérateur pseudodifférentiel analytique classique d'ordre 0 (formel pour l'instant), de symbole défini dans un voisinage de $\left.\Lambda_{\Phi_{0}}\right|_{\Omega}$ et donc aussi près de $\left.\Lambda_{\Phi_{\varepsilon}}\right|_{\Omega}$, si $0 \leqq \varepsilon<\varepsilon_{0}$, avec $\varepsilon_{0}$ assez petit.

Utilisant le fait que $\Phi_{0}$ est strictement convexe, et que le symbole principal $p$ de $P$ reste réel sur $A_{\Phi_{0}}$, on voit que l'on peut construire $e^{-t t P / h}$ formellement sous la forme:

$$
E_{t} u(x, h)=(2 \pi h)^{-n} \iint e^{i(\varphi(t, x, \eta)-v \cdot \eta) / h} a(i, x, \eta, h) u(y, h) d y d \eta .
$$

Ici $\varphi$ vérifie l'équation de Hamilton-Jacobi standard:

$$
\partial_{t} \varphi+p\left(x, \partial_{x} \varphi\right)=0, \varphi_{\mid t=0}=x \cdot \xi,
$$

et a s'obtient par résolution des équations de transport standard, avec condition initiale $a_{\mid t=0}=1$. Ainsi $\varphi$ est une fonction holomorphe et a est un symbole analytique classique d'ordre 0 défini dans un voisinage de: $\{(t, x, \eta) ; t \in \mathbb{R}, \exists(y, \xi)$ $\left.\operatorname{avec}(x, \xi)=\exp \left(t H_{p}\right)(y, \eta),(y, \eta) \in K_{0}\right\}$. Pour $\left(y_{0}, \eta_{0}\right) \in K_{0}$, on va étudier le linéarisé $E_{t}\left(y_{0}, \eta_{0}\right)$ défini dans la Sect. 2 . 
Lemme 3.1. $E_{t}\left(y_{0}, \eta_{0}\right)$ est unitaire sur $H_{\Phi_{0}}\left(\mathbb{C}^{n}\right)$.

Ici les espaces sont définis avec $h=1$. (Il sera clair par le contexte si $H_{\Phi_{0}}$ désigne l'espace avec $h=1$ ou avec $h$ variable). Dans l'énoncé on utilise que $\Phi_{0}$ est déjà une forme quadratique. Sinon il faudrait remplacer les deux $\Phi_{0}$ par leurs Hessiens respectivement en $y_{0}$ et en $x(t)$ où

$$
(x(t), \xi(t))=\exp \left(t H_{p}\right)\left(y_{0}, \eta_{0}\right) .
$$

Démonstration. Soient $u=u_{h}, v=v_{h} \in H_{\Phi_{0}}(\Omega)$ des états concentrés près de $y_{0}$, au sens que:

$$
\|u\|_{\Phi_{0}, \Omega \backslash B\left(y_{0}, h^{a}\right)}=O\left(h^{\infty}\right)\|u\|_{\Phi_{0}, \Omega},
$$

et de même pour $v$. (Voir aussi plus loin dans la démonstration.) Ici $1 / 3<a<1 / 2$. Alors à l'aide d'une réalisation standard de $E_{t}$, on sait définir $E_{t} u$, $E_{t} v \in H_{\Phi_{0}}\left(B\left(x(t), \varepsilon_{0}\right)\right)$, pour $|t| \leqq T_{0}, \varepsilon_{0}>0$. De même si on choisit une réalisation de $P$ (voir [8]), on obtient $\left(D_{t}-P\right) E_{t} u=O\left(h^{\infty}\right)\|u\|_{\Phi_{0}}$ dans $H_{\Phi_{0}}\left(B\left(x(t), \varepsilon_{0}\right)\right)$, et $E_{t} u$ est concentré près de $x(t)$ et de même pour $E_{t} v$. Alors modulo $O\left(h^{\infty}\right)\|u\|_{\Phi_{0}, \Omega}\|v\|_{\Phi_{0}, \Omega}$, on trouve:

$$
h D_{t}\left(E_{t} u \mid E_{t} v\right)_{\Phi_{0}, B\left(x(t), \varepsilon_{0}\right)}=-\left(P E_{t} u \mid E_{t} v\right)+\left(E_{t} u \mid P E_{t} v\right) .
$$

Comme $P$ est formellement autoadjoint dans $H_{\Phi_{0}}$, et $E_{t} u, E_{t} v$ sont concentrés près de $x(t)$, on montre (comme par exemple dans [8]) que $\left(P E_{t} u \mid E_{t} v\right) \equiv\left(E_{t} u \mid P E_{t} v\right)$, d'où: $D_{t}\left(E_{t} u \mid E_{t} v\right) \equiv 0$ ce qui donne

$$
\left(E_{t} u \mid E_{t} v\right) \equiv(u \mid v)
$$

localement uniformément par rapport à $t$.

Soit maintenant $\Psi_{0}$ une deuxième forme quadratique st.pl.s.h. telle que $\Phi_{0}-\Psi_{0} \sim|x|^{2}$. Si $\tilde{u} \in H_{\Psi_{0}}\left(\mathbb{C}^{n}\right)(\operatorname{avec} h=1)$, alors $\tilde{v}_{t}=E_{t}\left(y_{0}, \eta_{0}\right) \tilde{u} \in H_{\Psi_{t}}\left(\mathbb{C}^{n}\right)$, où $\Psi_{t}$ a les mêmes propriétés que $\Psi_{0}$, et $\Lambda_{\Psi_{t}}=d\left(\exp \left(t H_{p}\right)\right)\left(\Lambda_{\psi_{0}}\right)$. Pour revenir à $H_{\Phi_{0}}(\Omega)$, on fait les mêmes substitutions que dans la Sect. 2:

$$
u(x, h)=W\left(y_{0}, \eta_{0}\right) \tilde{u}(x)=h^{-n} e^{\left(i\left(x-y_{0}\right) \eta_{0}+\Phi_{0}\left(y_{0}\right)\right) / h} \tilde{u}(\tilde{x}), x=x_{0}+h^{1 / 2} \tilde{x} .
$$

Alors,

$$
\|u\|_{\Psi_{v_{0}}, \mathbb{C}^{n}}=\|\tilde{u}\|_{\Psi_{0}, \mathbb{C}^{n}},
$$

où la norme à gauche est avec $h$ variable et la norme à droite avec $h=1$. Ici on pose:

$$
\begin{gathered}
\Psi_{y_{0}}(x)=\Phi_{0}\left(y_{0}\right)+\left\langle\nabla \Phi_{0}\left(y_{0}\right),\left(x-y_{0}\right)\right\rangle+\Psi_{0}(x-y), \text { et on } a: \\
\Phi_{0}(x)-\Psi_{y_{0}}(x) \sim\left|x-y_{0}\right|^{2} .
\end{gathered}
$$

On en déduit que:

$$
\begin{gathered}
\|u\|_{\Phi_{0}, \Omega \backslash B\left(y_{0}, h^{a}\right)}=O\left(h^{\infty}\right)\|\tilde{u}\|_{\Psi_{0}, \mathbb{C}^{n}}, \\
\|u\|_{\Phi_{0}, B\left(y_{0}, h^{a}\right)}=\|\tilde{u}\|_{\Phi_{0}, \mathbb{C}^{n}}+O\left(h^{\infty}\right)\|\tilde{u}\|_{\Psi_{0}, \mathbb{C}^{n}}
\end{gathered}
$$

Ecrivons $W_{0}=W\left(y_{0}, \eta_{0}\right), W_{t}=W(x(t), \xi(t)), v_{t}=W_{t} \tilde{v}_{t}$. Alors $\left\|v_{t}\right\|_{\Psi_{x(t)}}=\left\|\tilde{v}_{t}\right\|_{\Psi_{t}}$, où $\Psi_{x(t)}=\Phi_{0}(x(t))+\left\langle\nabla \Phi_{0}(x(t)),(x-x(t)\rangle+\Psi_{t}(x-x(t))\right.$, et on a des relations analogues à (3.16), (3.17) pour $\Psi_{x(t)}, v_{t}, \tilde{v}_{t}$. 
Comme dans la Sect. 2, on voit que:

$$
\left\|E_{t} u-v_{t}\right\|_{\Phi_{0}, B\left(x(t), h^{a}\right)}=O\left(h^{3 a-1}\right)\|u\|_{\Phi_{0}, \Omega} .
$$

Combinant (3.14) avec $v=u,(3.17)$ et son analogue pour $v_{t}, \tilde{v}_{t}$, avec (3.18), on trouve dans la limite $h \rightarrow 0$ :

$$
\left\|E_{\tau}\left(y_{0}, \eta_{0}\right) \tilde{u}\right\|_{\Phi_{0}, \mathbb{C}^{n}}=\|\tilde{u}\|_{\Phi_{0}, \mathbb{C}^{n}}, \tilde{u} \in H_{\Psi_{0}}\left(\mathbb{C}^{n}\right) .
$$

Comme $H_{\Psi_{0}}\left(\mathbb{C}^{n}\right)$ est dense dans $H_{\Phi_{0}}\left(\mathbb{C}^{n}\right)$, on trouve que $E_{t}\left(y_{0}, \eta_{0}\right)$ est isométrique dans $H_{\Phi_{0}}\left(\mathbb{C}^{n}\right)$. Comme d'autre part $E_{t}\left(y_{0}, \eta_{0}\right)$ admet un inverse borné dans $L\left(H_{\Phi_{0}}\left(\mathbb{C}^{n}\right), H_{\Phi_{0}}\left(\mathbb{C}^{n}\right)\right)$, on trouve que $E_{t}\left(y_{0}, \eta_{0}\right)$ est unitaire.

Pour $0<\varepsilon<\varepsilon_{0}$ avec $\varepsilon_{0}$ assez petit, on s'intéresse à l'action de $E_{t}$ dans l'espace $H_{\Phi_{\varepsilon}}$ suffisament près de $\Pi_{x} K_{0}, t \geqq 0$. Comme déjà rappelé au début de la Sect. 2 , si $A$ est donné par (2.1) et si $\Phi_{1}$ et $\Phi_{2}$ sont des fonctions pl.s.h. de classe $C^{2}$ définies dans des voisinages de $y_{0}$ et $x_{0}$ respectivement avec $\left(x_{0}, \xi_{0}\right) \in \Lambda_{\Phi_{2}},\left(y_{0}, \eta_{0}\right) \in \Lambda_{\Phi_{1}}$, et:

$$
\Lambda_{\Phi_{2}}=H_{A}\left(\Lambda_{\Phi_{1}}\right), \Phi_{2}\left(x_{0}\right)=-\operatorname{Im} \varphi\left(x_{0}, y_{0}, \theta_{0}\right)+\Phi_{1}\left(y_{0}\right),
$$

alors on peut réaliser $A$ comme un opérateur de norme $O(1): H_{\Phi_{1}}\left(\Omega_{1}\right) \rightarrow H_{\Phi_{2}}\left(\Omega_{2}\right)$, où $\Omega_{1}$ et $\Omega_{1}$ sont des petits voisinages de $y_{0}, x_{0}$. Dans le cas précédent, si $y_{0} \in \Pi_{x} K_{0}$, $\varepsilon>0$ petit, $t \geqq 0$, alors $E_{t}$ est $O(1)$ comme opérateur borné: $H_{\Phi_{t}}\left(\Omega_{0}^{\prime}\right) \rightarrow H_{\Phi_{\varepsilon, t}}\left(\Omega_{t}\right)$, où $\Omega_{0}^{\prime}$ est un petit voisinage de $x(t)$. Ici $\Phi_{\varepsilon, t}$ est donné par:

$$
\partial_{t} \Phi_{\varepsilon, t}=\operatorname{Im} p\left(x, 2 / i \partial_{x} \Phi_{\varepsilon, t}\right), \Phi_{\varepsilon, 0}=\Phi_{\varepsilon} .
$$

On a aussi

$$
\Lambda_{\Phi_{\varepsilon, t}}=\exp \left(t H_{p}\right)\left(\Lambda_{\Phi_{\varepsilon}}\right)
$$

D'autre part, grâce à (1.5) on a $\operatorname{Im} p\left(x,(2 / i) \partial_{x} \Phi_{\varepsilon, t}\right) \sim-\operatorname{dist}\left(x, \Pi_{x} \Sigma\right)^{2}$ pour $t=0$, si $\varepsilon>0$ est assez petit. Utilisant (3.22) et le fait que $p$ est constant le long des trajectoires de $H_{p}$, on voit que ceci reste vrai pour $t>0$, ce qui donne:

$$
\Phi_{\varepsilon}-\Phi_{\varepsilon . t} \sim \operatorname{dist}\left(\cdot, \Pi_{x} \Sigma\right)^{2} .
$$

(Comme nous savons de [8], cette relation empêche l'apparition de caustiques dans un petit voisinage $\Omega_{t}$ de $x(t)$, et les arguments ci-dessus fonctionnent pour $0 \leqq t<+\infty$.) Ceci entraine que (2.6) est vérifié pour $\Phi_{1}=\Phi_{2}=\Phi_{\varepsilon}, \Sigma_{1}=\Sigma_{2}=\Sigma$. On pourra donc appliquer le resultat de la Sect. 2 .

On a déjà vu que $E_{t}\left(y_{0}\right)=E_{t}\left(y_{0}, \eta_{0}\right)$ est unitaire $H_{\Phi_{0}}\left(\mathbb{C}^{n}\right) \rightarrow H_{\Phi_{0}}\left(\mathbb{C}^{n}\right)$, et on s'intéresse maintenant à sa norme $H_{\Phi_{\varepsilon, 0}^{\prime \prime}} \rightarrow H_{\Phi_{\varepsilon, x(t)}^{\prime \prime}}$ où $\Phi_{\varepsilon, 0}^{\prime \prime}$ désigne le Hessien de $\Phi_{\varepsilon}$ en $y_{0}$, quand $\left(y_{0}, \eta_{0}\right) \in K_{0}$.

Pour chaque $x_{0} \in \Pi_{x} K_{0}$, on peut trouver un opérateur intégral de Fourier à phase quadratique et amplitude constante qui réalise un opérateur unitaire:

$$
U\left(x_{0}\right): H_{\Phi_{0}}\left(\mathbb{C}^{n}\right) \rightarrow H_{\Phi^{\prime}\left(x_{0}\right)}\left(\mathbb{C}_{x^{\prime}}^{n-d}\right) \otimes L^{2}\left(\mathbb{R}_{x^{\prime \prime}}^{d}\right),
$$

tel que la transformation canonique associée, $H_{x_{0}}$ envoie $T_{\left(x_{0}, \xi_{0}\right)} \Sigma$ sur $\Lambda_{\Phi_{0}^{\prime}} \otimes\left\{x^{\prime \prime}=\xi^{\prime \prime}=0\right\}, \quad T_{\left(x_{0}, \xi_{0}\right)} \sum^{\mathbb{C}}$ sur $\mathbb{C}_{x^{\prime}, \xi^{\prime}}^{2(n-d)} \otimes\left\{x^{\prime \prime}=\xi^{\prime \prime}=0\right\}, \quad N_{+,\left(x_{0}, \xi_{0}\right)}$ sur $\mathbb{R}_{x^{\prime \prime}}^{d}$, $N_{-,\left(x_{0}, \xi_{0}\right)}$ sur $\mathbb{R}_{\xi^{\prime \prime}}^{d}$. Ici $\Phi^{\prime}\left(x_{0}\right)$ est une forme quadratique strictement plurisousharmonique. Plus précisement on peut obtenir $U\left(x_{0}\right)$ comme le composé de:

$1^{\circ}$ : Une transformation de Bargman inverse (globale avec $h=1$ ):

$$
H_{\Phi_{0}}\left(\mathbb{C}^{n}\right) \rightarrow L^{2}\left(\mathbb{R}^{n}\right)
$$


$2^{\circ}$ : Un opérateur métaplectique standard $L^{2}\left(\mathbb{R}^{n}\right) \rightarrow L^{2}\left(\mathbb{R}^{n}\right)$, tel que la transformation canonique associée au composé des deux opérateurs $1^{\circ}$ et $2^{\circ}$ envoie: $T_{\left(x_{0}, \xi_{0}\right)} \Sigma$ $\operatorname{sur} \mathbb{R}_{\left(x^{\prime}, \xi^{\prime}\right)}^{2 n} \otimes\left\{x^{\prime \prime}=\xi^{\prime \prime}=0\right\}, N_{+.\left(x_{0}, \xi_{0}\right)}$ sur $\mathbb{R}_{x^{\prime \prime},}^{d}, N_{-,\left(x_{0}, \xi_{0}\right)}$ sur $\mathbb{R}_{\xi^{\prime \prime}}^{d}$

$3^{\circ}$ : Une transformation de Bargman partielle dans les variables $x^{\prime}$.

(On réalise $U\left(x_{0}\right)$ par un choix de bon contour, si $u \in H_{\Phi_{1}}$, avec $\Phi_{1}-\Phi_{0} \sim|x|^{2}$, et on définit ensuite $U\left(x_{0}\right)$ sur $H_{\Phi_{0}}$ par densité.) Si on utilise la phase et le symbole comme paramètres, on peut en plus s'arranger pour que $U\left(x_{0}\right)$ varie dans un ensemble borné, quand $x_{0}$ varie dans $\Pi_{x} K_{0}$.

Lemme 3.2. Pour $\varepsilon>0$ assez petit, $N_{ \pm, \varrho}^{\mathbb{C}}$ est strictement positif /négatif par rapport à $T_{\varrho}\left(\Lambda_{\varepsilon G}\right)$, pour tout $\varrho \in K_{0}$. Plus précisément $\pm(1 / i) \sigma(u, \bar{u})>0$ pour tout $u \neq 0$, $u \in N_{ \pm . \varrho}^{\mathbb{C}}$, où $\bar{u}$ désigne le vecteur complexe conjugué par rapport au sous espace totalement réel de dimension maximale $T_{\varrho}\left(\Lambda_{\varepsilon G}\right)$.

Démonstration. Modulo $O\left(\varepsilon^{2}\right)$, on peut identifier $T_{\varrho}\left(\Lambda_{\varepsilon G}\right)$ avec $\exp \left(i \varepsilon H_{G^{\prime \prime}}\right)\left(T_{\varrho} \mathbb{R}^{2 n}\right)$. Etudions d'abord la positivité/négativité de $N_{ \pm, \varrho}^{\mathbb{C}}$ par rapport à $\exp \left(i \varepsilon H_{G^{\prime \prime}}\right)\left(T_{\varrho} \mathbb{R}^{2 n}\right)$. Ceci revient à étudier la positivité/négativité de $\exp \left(-i \varepsilon H_{G^{\prime \prime}}\right)\left(N_{ \pm, \varrho}^{\mathbb{C}}\right)$ par rapport à $T_{\varrho} \mathbb{R}^{2 n}$. Un vecteur typique de ces deux espaces s'écrit: $u=v-i \varepsilon F_{G}(v)+O\left(\varepsilon^{2}\|v\|\right), v \in N_{ \pm, o}^{\mathbb{C}}$, où $F_{G}$ est la matrice fondamentale de $G$ définie par $G^{\prime \prime}(t, s)=\sigma\left(t, F_{G} s\right)$. Modulo $O\left(\varepsilon^{2}\|u\|^{2}\right)$, on obtient alors: $\sigma(u, \bar{u})$ $=\sigma\left(v-i \varepsilon F_{G} v, \bar{v}+i \varepsilon F_{G} \bar{v}\right) \equiv 2 \varepsilon i \sigma\left(v, F_{G} \bar{v}\right)=2 \varepsilon i G^{\prime \prime}(v, \bar{v})$, puisque $N_{ \pm, \varrho}$ sont isotropes. Comme $G^{\prime \prime}$ est positive/négative sur $N_{ \pm, \varrho}$, on obtient:

$$
\pm i^{-1} \sigma(u, \bar{u}) \geqq \varepsilon\|u\|^{2} / C_{0}, u \in \exp \left(-i \varepsilon H_{G^{\prime \prime}}\right)\left(N_{ \pm, \varrho}^{\mathbb{C}}\right),
$$

ce qui suffit pour justifier notre approximation initiale et démontrer le lemme.

Considérons ensuite $H_{x_{0}}\left(\Lambda_{\Phi_{\varepsilon, x_{0}}^{\prime \prime}}\right)=L_{x_{0}}$. On sait d'une part que l'espace $H_{x_{0}}\left(T_{\left(x_{0}, \xi_{0}\right)} \Sigma\right)=\Lambda_{\Phi_{0}^{\prime}} \otimes\left\{x^{\prime \prime}=\xi^{\prime \prime}=0\right\}$ est contenu dans l'espace $I$-lagrangien $L_{x_{0}}$, et d'autre part que $L_{x_{0}} \cap \mathbb{C}_{\xi^{\prime \prime}}^{d}=\{0\}$ car $\mathbb{C}_{\xi^{\prime \prime}}^{d}$ est strictement négatif par rapport à $L_{x_{0}}$. Si $(0, \xi) \in L_{x_{0}}$, on $a: \operatorname{Im} \sigma\left(\left(y^{\prime}, 0, \eta^{\prime}, 0\right),(0, \xi)\right)=-\operatorname{Im} y^{\prime} \cdot \xi^{\prime}=0$, pour tout $\left(y^{\prime}, \eta^{\prime}\right) \in \Lambda_{\Phi_{0}^{\prime}}$, ce qui entraine que $\xi^{\prime}=0$. Donc $\mathbb{C}_{\xi}^{n} \cap L_{x_{0}}=\{0\}$, et $L_{x_{0}}$ est de la forme $\Lambda_{\psi}$, où $\psi(x)=\Phi^{\prime}\left(x_{0}\right)\left(x^{\prime}\right)+D\left(x_{0}\right)\left(x^{\prime \prime}\right)$. ( $\psi$ depend bien sûr aussi de $x_{0}$.) D'après le Lemme 3.2 , on sait que $\mathbb{C}_{\xi^{\prime}}^{d}$ est strictement négatif par rapport à $\Lambda_{4}$ et donc aussi par rapport à $\Lambda_{D\left(x_{0}\right)}\left(\right.$ dans $\left.\mathbb{C}^{2 d}\right)$. D'après [8], on sait alors que $D\left(x_{0}\right)$ est strictement plurisousharmonique. De même, la positivité de $\mathbb{C}_{x^{\prime \prime}}^{d}$, montre que $D\left(x_{0}\right)$ est strictement convexe:

$$
D\left(x_{0}\right)\left(x^{\prime \prime}\right) \sim\left|x^{\prime \prime}\right|^{2}
$$

Comme dans [8, Chap. 3], on voit ensuite que $U\left(x_{0}\right)$ est bien défini comme opérateur borné: $H_{\Phi_{\varepsilon, x_{0}}^{\prime \prime}} \rightarrow H_{\psi_{x_{0}}}$ avec un inverse borné (le tout uniformément par rapport à $x_{0} \in \Pi_{x} K_{0}$ ). Modulo un facteur indépendant de $t$ et de $y_{0}$, notre problème est alors de majorer la norme de:

$$
F_{t}\left(y_{0}\right)=U\left(x_{0}\right) E_{t}\left(y_{0}\right) U\left(x_{0}\right)^{-1}: H_{\psi_{y_{0}}} \rightarrow H_{\psi_{x_{0}}},
$$

sachant que cet opérateur est unitaire dans $H_{\Phi_{0}^{\prime}}\left(\mathbb{C}^{n-d}\right) \otimes L_{x^{\prime \prime}}^{2}$. Il est clair que $F_{t}\left(y_{0}\right)$ peut s'écrire sous la forme:

$$
F_{t}\left(y_{0}\right) u(x)=(2 \pi)^{d-n} \iint e^{i\left(\varphi\left(t, x^{\prime}, y^{\prime}, \theta^{\prime}\right)+\left(A_{t} x^{\prime \prime}-y^{\prime \prime}\right) \theta^{\prime \prime}\right)} a_{t} u(y) d y d \theta .
$$


Modulo conjuguaison par les transformations canoniques associées à $U\left(x_{0}\right)$ et $U\left(y_{0}\right)$, la phase $\varphi$ décrit $d\left(\exp \left(t H_{p}\right)\right): T_{\left(y_{0}, \eta_{0}\right)} \Sigma \rightarrow T_{\left(x_{0}, \xi_{0}\right)} \Sigma$ et la phase $\left(A_{t} x^{\prime \prime}-y^{\prime \prime}\right) \theta^{\prime \prime}$ décrit la partie transversale: $d\left(\exp \left(t H_{p}\right)\right): T_{\left(v_{0}, \eta_{0}\right)} \Sigma^{\sigma} \rightarrow T_{\left(x_{0}, \xi_{0}\right)} \Sigma^{\sigma}$. Dans les coordonnées $\left(x^{\prime \prime}, \xi^{\prime \prime}\right)$ cette dernière transformation devient $\left(A_{t} x^{\prime \prime}, \xi^{\prime \prime}\right) \rightarrow\left(x^{\prime \prime},{ }^{t} A_{t} \xi^{\prime \prime}\right)$, et comme le flot est expansif sur $N_{+}$et contractif sur $N_{-}$, on obtient:

$$
\left\|A_{t}\right\| \leqq C_{0} e^{-t / C_{0}}, t \geqq 0 \text {, pour un } C_{0} \text { indépendant de } y_{0} .
$$

D'après la définition de $d_{0}$, on a aussi:

$$
\left.\left|\operatorname{det} A_{t}\right| \leqq C(d) e^{-d t}, t \geqq 0, \text { pour tout } d \in\right] 0, d_{0}[\text {. }
$$

Si $V$ est unitaire dans $H_{\Phi^{\prime}\left(x_{0}\right)}\left(\mathbb{C}^{n-d}\right)$, alors $V \otimes I_{x^{\prime \prime}}$ est unitaire à la fois dans $H_{\Phi^{\prime}\left(x_{0}\right)} \otimes L^{2}$ et dans $H_{\psi_{0}}$. Il suffit alors de majorer la norme de:

$$
\left(V \otimes I_{x^{\prime \prime}}\right) \circ F_{t}\left(y_{0}\right): H_{\psi, y_{0}} \rightarrow H_{\psi, x(t)},
$$

sachant que cet opérateur est unitaire de $H_{\Phi^{\prime}\left(y_{0}\right)} \otimes L^{2} \rightarrow H_{\Phi^{\prime}\left(x_{0}\right)} \otimes L^{2}$. Avec un choix convenable de $V$, on peut se ramener au cas où $\varphi\left(t, x^{\prime}, y^{\prime}, \theta^{\prime}\right)=\left(x^{\prime}-y^{\prime}\right) \theta^{\prime}$, et on trouve alors le nouvel opérateur que l'on notera encore $F_{t}\left(y_{0}\right)$ :

$$
F_{t}\left(y_{0}\right) u(x)=a_{t} u\left(x^{\prime}, A_{t} x^{\prime \prime}\right) .
$$

L'unitarité dans $H_{\Phi_{0}^{\prime}} \otimes L^{2}$, entraine que:

$$
\left|a_{t}\right|=\left|\operatorname{det} A_{t}\right|^{1 / 2} \leqq C(d)^{1 / 2} e^{-d t / 2}, \quad t \geqq 0,
$$

pour $0<d<d_{0}$. La variable $x^{\prime}$ est maintenant devenue un paramètre muet et peut être oubliée dans les calculs. Par une formule de la moyenne convenable, on montre que:

$$
\left|u\left(x^{\prime \prime}\right)\right| \leqq C\|u\|_{D\left(y_{0}\right)} e^{D\left(y_{0}\right)\left(x^{\prime \prime}\right)}, \forall u \in H_{D\left(\gamma_{0}\right)}\left(\mathbb{C}^{d}\right) .
$$

Pour $t \geqq t_{0} \gg 0$, on a $D(x(t))\left(x^{\prime \prime}\right)-D\left(y_{0}\right)\left(A_{t} x^{\prime \prime}\right) \geqq C_{0}^{-1}\left|x^{\prime \prime}\right|^{2}$, et donc (en négligeant $\left.x^{\prime}\right)$ :

$$
\begin{gathered}
\left\|F_{t}\left(y_{0}\right)\right\|_{D(x(t))}^{2}=\left|a_{t}\right|^{2} \int\left|u\left(A_{t} x^{\prime \prime}\right)\right|^{2} e^{-2 D\left(x_{0}\right)\left(x^{\prime \prime}\right)} L\left(d x^{\prime \prime}\right) \\
\leqq C^{2}\left|a_{t}\right|^{2}\|u\|_{D\left(y_{0}\right)}^{2} \int e^{-2\left(D(x(t))\left(x^{\prime \prime}\right)-D\left(y_{0}\right)\left(A_{t} x^{\prime \prime}\right)\right)} L\left(d x^{\prime \prime}\right) \leqq C^{\prime}\left|a_{t}\right|^{2}\|u\|_{D\left(y_{0}\right)}^{2} .
\end{gathered}
$$

Utilisant aussi (3.31), nous avons montré:

Proposition 3.3. Il existe $\varepsilon_{0}>0$ tel que pour $\left.0<\varepsilon<\varepsilon_{0}, d \in\right] 0, d_{0}[$ :

$$
\left\|E_{t}\left(y_{0}\right)\right\|_{L\left(\Phi_{\varepsilon, 2}^{\prime \prime}, \Phi_{c}^{\prime \prime}, x(t)\right)} \leqq C(\varepsilon, d) e^{-d t / 2}, \quad t \geqq 0, \text { pour tout } y_{0} \in \Pi_{x} K_{0} .
$$

Il est facile de montrer que $\left\|E_{t}\left(y_{0}\right)\right\|$ varie continument avec $t$ et $\left(y_{0}, \eta_{0}\right) \in \Sigma$, ce qui entraine que (3.34) est encore valable quand $\left(y_{0}, \eta_{0}\right) \in \Sigma$ est dans un voisinage de $K_{0}$ dependant de $t$. Combinant ceci avec la Proposition 2.1, on obtient:

Proposition 3.4. Soit \& fixé assez petit. Alors pour tout $d \in] 0, d_{0}[$, il existe $C(d)>0$ et pour tout $t>0$, il existe un voisinage $\Omega_{t, d}$ de $\Pi_{x} K_{0}$, qui décroit quand t augmente, tels que pour tout $W \subset \Omega_{t, d}$, on a:

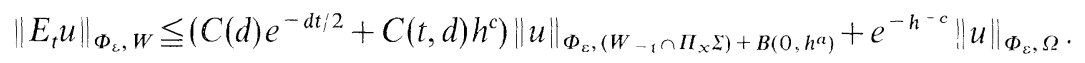

Ici $W_{-t}=\Pi_{x} \exp \left(-t H_{p}\right)\left(\Pi_{x}^{-1} W \cap \Lambda_{\Phi_{0}}\right)$. 


\section{Fin de la démonstration}

Soit $G$ la même fonction qu'avant. On peut alors trouver un voisinage ouvert $\Omega$ cC $T^{*} \mathbb{R}^{n}$ de $K_{0}$ avec les propriétés suivantes:

$$
\begin{gathered}
|\nabla G| \sim H_{p} G \geqq 0 \text { dans } \Omega . \\
H_{p} G+|p|>0 \text { dans } C \Omega .
\end{gathered}
$$

Si on a identifié $\Omega$ avec un ouvert de $\mathbb{C}^{n}$ comme dans la Sect. 2, alors pour $\varepsilon$ assez petit, on $a$ :

$$
\begin{gathered}
\left.\operatorname{Im} p\right|_{\Lambda_{\Phi_{r}}} \leqq 0 \text { sur } \Omega . \\
\left.p\right|_{\Lambda \Phi_{r}} \neq 0 \text { sur } \partial \Omega .
\end{gathered}
$$

Soient $\Omega_{2} \subset \subset \Omega_{1} \subset \subset \Omega$ deux autres ouverts, tels que (4.4) soit valable sur $\operatorname{Ad}\left(\Omega \backslash \Omega_{2}\right)$ pour $\varepsilon$ assez petit, et choisissons une réalisation de $P$ à contour singulier de norme $O(1): H_{\Phi_{\varepsilon}}(\Omega) \rightarrow H_{\Phi_{\varepsilon}}\left(\Omega_{1}\right)$ pour $0 \leqq \varepsilon \leqq \varepsilon_{1}, \varepsilon_{1}$ assez petit. L'étape principale sera de montrer (pour tout $\varepsilon>0$ fixé, suffisament petit):

Proposition 4.1. Pour tout $d \in] 0, d_{0}[$, il existe $C>0$ tel que:

$$
\|u\|_{\Omega_{2}} \leqq C\left(h^{-1}\|(P-z) u\|_{\Omega_{1}}+\|u\|_{\Omega \backslash \Omega_{2}}\right)
$$

pour tout $u \in H_{\Phi_{\varepsilon}}(\Omega), z \in[-1 / C, 1 / C]-i[0, d h / 2]$.

(Ici et dans la suite, les normes sont celles de $H_{\Phi_{\varepsilon}}$, si rien d'autre n'est indiqué.)

Démonstration. Dans la suite on restreint $z$ à $\left[-\varepsilon_{0} / 2, \varepsilon_{0} / 2\right]-i[0, d h / 2]$ pour un $\varepsilon_{0}$ assez petit. Soit $K$ l'ensemble des trajectoires captées dans $p^{-1}\left(\left[-\varepsilon_{0}, \varepsilon_{0}\right]\right), K=\Gamma_{+}$ $\cap \Gamma_{-}$, où $\Gamma_{ \pm}$est l'ensemble des points $\varrho \in p^{-1}\left(\left[-\varepsilon_{0}, \varepsilon_{0}\right]\right)$ tels que $\exp \left(t H_{p}\right)(\varrho)$ ne tend pas vers l' $\infty$ quand $-t$ tend vers $\pm \infty$. (Voir l'appendice de [4].) Pour $\varepsilon_{0}>0$ et $h>0$ assez petits, on sait alors que $(p-z) \mid \Lambda_{\Phi_{\varepsilon}}$ est elliptique au dessus de $\Omega \backslash \Omega_{2}$, uniformément par rapport à $z$.

Soit $x_{0} \in \operatorname{Ad}\left(\Omega_{2}\right), \xi_{0}=(2 / i) \partial_{x} \Phi_{0}\left(x_{0}\right)$. On va estimer la norme de $u$ sur un voisinage de $x_{0}$, en distinguant différents cas:

1) $\left|p\left(x_{0}, \xi_{0}\right)\right| \geqq \varepsilon_{0}$ ou $H_{p} G\left(x_{0}, \xi_{0}\right)>0$. Alors $(p-z) \mid \Lambda_{\Phi_{\varepsilon}}$ est elliptique en $x_{0}$, et on obtient:

$$
\|u\|_{V} \leqq C\left(\|(P-z) u\|_{\Omega_{1}}+h^{2}\|u\|_{\Omega}\right)
$$

où $V$ est un voisinage de $x_{0}$.

2) $\left|p\left(x_{0}, \xi_{0}\right)\right| \leqq \varepsilon_{0}$ et $H_{p} G\left(x_{0}, \xi_{0}\right)=0$. Soit alors $T\left(x_{0}, \xi_{0}\right) \in[0,+\infty]$ le plus grand des $T \geqq 0$ tels que $H_{p} G\left(\exp \left(-s H_{p}\right)\left(x_{0}, \xi_{0}\right)\right)=0$ pour $0 \leqq s \leqq T$. On ne peut avoir $T\left(x_{0}, \xi_{0}\right)=+\infty$, que si $\left(x_{0}, \xi_{0}\right) \in \Gamma_{+}$.

2a) $T\left(x_{0}, \xi_{0}\right)=+\infty$. Soit $0<T<+\infty$. Pour $0 \leqq t \leqq T$, on peut alors définir $E_{t, z} u(x)=e^{i t z / h} E_{t} u(x)$, dans un voisinage $V_{0}$ de $x_{0}$, et on obtient:

$$
\left\|E_{t, z} u\right\|_{V_{0}} \leqq C(T)\|u\|_{V_{-1}},
$$

où $V_{-t}$ est un petit voisinage de $\Pi_{x} \exp \left(-t H_{p}\right)\left(x_{0}, \xi_{0}\right)$. On montre aussi que:

$$
\left\|h D_{t} E_{t, z} u+E_{t, z}(P-z) u\right\|_{V_{0}}=O\left(h^{\infty}\right)\|u\|_{\Omega} .
$$


On pose $R_{T} v=i h^{-1} \int_{0}^{T} E_{t, z} v d t$. Alors, dans $V_{0}$, on $a$ :

$$
R_{T}(P-z) u=u-E_{T, z} u+i h^{-1} \int_{0}^{T}\left(h D_{t} E_{t, z} u+E_{t, z}(P-z) u\right) d t .
$$

Combinant (4.7)-(4.9) et la définition de $R_{T}$, on obtient:

$$
\left\|u-E_{T, z} u\right\|_{V_{0}} \leqq C(T) h^{-1}\|(P-z) u\|_{\Omega_{1}}+O_{T}\left(h^{\infty}\right)\|u\|_{\Omega},
$$

ce qui donne:

$$
\|u\|_{V_{0}} \leqq C(T)\left(h^{-1}\|(P-z) u\|_{\Omega_{1}}+\|u\|_{V_{-T}}+h^{2}\|u\|_{\Omega}\right) .
$$

Ici $V_{0}$ est un petit voisinage de $x_{0}$ et $V_{-T}$ un petit voisinage de $\Pi_{x} \exp \left(-T H_{p}\right)\left(x_{0}, \xi_{0}\right)$, que l'on pourra prendre arbitrairement petit, quitte à choisir $V_{0}$ assez petit.

2b) $T\left(x_{0}, \xi_{0}\right)<+\infty$. On obtient alors (4.11) avec $T=T\left(x_{0}, \xi_{0}\right)$, mais on veut dans ce cas estimer $\|u\|_{V_{-T}}$. On peut alors supposer que $T\left(x_{0}, \xi_{0}\right)=0$. Alors pour $T>0$ petit, on $a:\left\|E_{T, z} u\right\|_{\Phi_{\varepsilon, T}, V_{0}} \leqq\|u\|_{\Phi_{\ell}, \Omega}$, où $\Phi_{\varepsilon, T}$ est donné par (3.21). On a $\Phi_{\varepsilon, T}(x) \leqq \Phi_{\varepsilon}(x)$. Montrons que cette inégalité est stricte pour $x=x_{0}$, et supposons par l'absurde que $\Phi_{\varepsilon, T}\left(x_{0}\right)=\Phi_{\varepsilon}\left(x_{0}\right)$. Alors $\Phi_{\varepsilon, t}\left(x_{0}\right)=\Phi_{\varepsilon}\left(x_{0}\right), 0 \leqq t \leqq T$, et donc $(2 / i) \partial_{x} \Phi_{\varepsilon, t}\left(x_{0}\right)=(2 / i) \partial_{x} \Phi_{\varepsilon}\left(x_{0}\right)=\xi_{0}$. Alors d'après (3.22) on $a$ :

$$
\exp \left(-t H_{p}\right)\left(x_{0}, \xi_{0}\right) \in \Lambda_{\Phi_{\varepsilon}}, \quad 0 \leqq t \leqq T,
$$

et donc $\nabla \Phi_{\varepsilon}=\nabla \Phi_{0}$ le long de cette trajectoire. Ceci est impossible car $\nabla \Phi_{\varepsilon}-\nabla \Phi_{0}$ $=\varepsilon G+O\left(\varepsilon^{2}|\nabla G|^{2}\right)$, n'est pas identiquement nul en $\exp \left(-t H_{p}\right)\left(x_{0}, \xi_{0}\right)$ pour $\emptyset \leqq t \leqq T$.

Si $V_{0}$ est assez petit, on obtient donc:

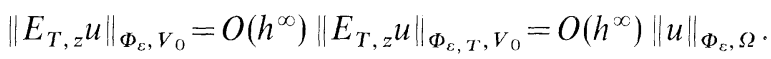

Comme (4.10) est encore valable, on obtient:

$$
\|u\|_{V_{0}} \leqq C\left(h^{-1}\|(P-z) u\|_{\Omega}+h^{2}\|u\|_{\Omega}\right) .
$$

Par un argument de recouvrement, on obtient:

Lemme 4.2. Pour tout voisinage $W$ de $K$ dans $p^{-1}\left(\left[-\varepsilon_{0}, \varepsilon_{0}\right]\right)$, il existe $C>0$ tel que:

$$
\|u\|_{\Omega_{2}} \leqq C\left(h^{-1}\|(P-z) u\|_{\Omega_{1}}+\|u\|_{W}+h^{2}\|u\|_{\Omega}\right),
$$

pour tout $u \in H_{\Omega_{\varepsilon}}(\Omega), z \in\left[-\varepsilon_{0} / 2, \varepsilon_{0} / 2\right]-i[0, d h / 2]$. Si $V$ est un voisinage de $\Gamma_{+}$dans $\operatorname{Ad}\left(\Omega_{2}\right)$, il existe $C>0$ tel que:

$$
\|u\|_{\Omega_{2} \backslash V} \leqq C\left(h^{-1}\|(P-z) u\|_{\Omega_{1}}+h^{2}\|u\|_{\Omega}\right),
$$

pour les mêmes $u, z$.

Il s'agit maintenant de se débarrasser du deuxième terme du membre de gauche de (4.13) et c'est ici que l'on doit utiliser la Proposition 3.4. Il nous faut d'abord un lemme géométrique:

Lemme 4.3. On se place dans $p^{-1}\left(\left[-\varepsilon_{0}, \varepsilon_{0}\right]\right)$. Pour lout $t>0$, on peut trouver un voisinage arbitrairement petit $W$ de $K$, et un $\varepsilon=\varepsilon(W)>0$, tels que: $\left(\exp \left(-t H_{p}\right)(W)\right.$ $+B(0, \varepsilon)) \backslash W$ soit disjoint de $\Gamma_{+}+B(0, \varepsilon)$ dans $p^{-1}\left(\left[-\varepsilon_{0}, \varepsilon_{0}\right]\right)$. 
Démonstration. Soit $W^{\prime}$ un voisinage ouvert arbitrairement petit de $K$. Soit $G$ une fonction fuite telle que $H_{p} G \geqq 0$, avec inégalité stricte pour $|G| \geqq 1$. Alors $K$ $C\{|G|<1\}$. Soit $v=\chi(x, \xi) H_{p}$, où $0 \leqq \chi \in C_{0}^{\infty}, \chi=1$ dans un grand voisinage de $K$. Alors $G_{T}=G \circ \exp (T v)$, est une nouvelle fonction fuite avec les mêmes propriétés que $G$. De plus si $T>0$ est assez grand, on $a$ :

$$
\left\{G_{T} \leqq 1\right\} \cap \Gamma_{+} \subset W^{\prime} .
$$

On pose $W=W^{\prime} \cap\left\{G_{t}<1\right\}$. Alors $\exp \left(-t H_{p}\right)(W) \subset\left\{G_{T}<1-2 \delta\right\}$, pour un $\delta>0$. Si $\varepsilon>0$ est assez petit, et $\varrho \in\left(\exp \left(-t H_{p}\right)(W)+B(0, \varepsilon)\right) \backslash W$, on a $G_{T}(\varrho)<1-\delta$ et donc aussi $\varrho \notin W^{\prime}$. D’après (4.15), on peut alors minorer la distance de $\varrho$ à $\left\{G_{T} \leqq 1\right\} \cap \Gamma_{+}$ par une constante $>0$, et comme $G_{T}(\varrho)<1-\delta$, on peut faire de même pour la distance de $\varrho$ à $\left\{G_{T} \geqq 1\right\} \cap \Gamma_{+}$. Avec un nouvel $\varepsilon>0$ suffisamment petit, on obtient alors la conclusion du lemme.

On applique maintenant la Proposition 3.4. Soit $\left.d^{\prime} \in\right] d, d_{0}[$ et $t>0$, tel que $C\left(d^{\prime}\right) e^{-\left(d^{\prime} / 2+\operatorname{Im} z\right) t / h} \leqq 1 / 3$, pour $\operatorname{Im} z \geqq-d / 2$. On choisit ensuite $\varepsilon_{0}>0$ suffisamment petit pour qu'il existe $W$, voisinage de $K$ dans $p^{-1}\left(\left[-\varepsilon_{0}, \varepsilon_{0}\right]\right)$, tel que (3.35) soit valable. Quitte à diminuer $W$, on peut aussi supposer que la conclusion du Lemme $4.3 \mathrm{~s}$ 'applique. Comme avant, on restreint $\operatorname{Re} z$ à $\left[-\varepsilon_{0} / 2, \varepsilon_{0} / 2\right]$. Combinant (4.10), avec $V_{0}$ remplacé par $W$ et $t=T$, avec (3.35), on trouve pour $h$ assez petit:

$$
\|u\|_{W} \leqq C h^{-1}\|(P-z) u\|_{\Omega_{1}}+C h^{2}\|u\|_{\Omega}+(1 / 2)\|u\|_{W-\imath}+B\left(0, h^{a}\right),
$$

où $W_{-t}=\Pi_{x} \exp \left(-t H_{p}\right)\left(\Pi_{x}^{-1} W \cap \Lambda_{\Phi_{0}}\right)$.

D'autre part

$$
\begin{aligned}
& \|u\|_{W-t}+B\left(0, h^{a}\right) \leqq\|u\|_{W}+\|u\|_{\left(W-{ }_{t}+B\left(0, h^{a}\right) \backslash W\right) \cap p^{-1}\left(\left[-\varepsilon_{0}, \varepsilon_{0}\right]\right)} \\
& \left.+\|u\|_{(W-t}+B\left(0, h^{a}\right) \backslash W\right) \backslash p^{-1}\left(\left[-\varepsilon_{0}, \varepsilon_{0}\right]\right),
\end{aligned}
$$

et le dernier terme se majore ici par $C\left(\|(P-z) u\|_{\Omega_{1}}+h^{2}\|u\|_{\Omega}\right.$, comme on est dans la région elliptique. On obtient alors de (4.16) avec une nouvelle constante:

$$
\|u\|_{W} \leqq C\left(h^{-1}\|(P-z) u\|_{\Omega_{1}}+h^{2}\|u\|_{\Omega}+\|u\|_{p^{-1}\left(\left[-\varepsilon_{0} \cdot \varepsilon_{0}\right]\right) \cap\left(W{ }_{-t}+B\left(0 . h^{a}\right) \backslash W\right)}\right) .
$$

Comme $\left[\left(W_{-t}+B\left(0, h^{a}\right)\right) \backslash W\right] \cap p^{-1}\left(\left[-\varepsilon_{0}, \varepsilon_{0}\right]\right)$ est disjoint d'un voisinage de $\Gamma_{+}$, on peut appliquer (4.14), pour majorer le dernier terme de (4.17) et on obtient:

$$
\|u\|_{W} \leqq C\left(h^{-1}\|(P-z) u\|_{\Omega_{1}}+h^{2}\|u\|_{\Omega}\right) .
$$

La substitution dans (4.13) donne la Proposition 4.1.

Pour terminer la démonstration du Théorème, on retourne à la situation avant la transformation de F.B.I. $P$ désigne l'opérateur initial, et $Q$ son image par la transformation de F.B.I., $U$. Dans (3.7) et (3.9), on pourra remplacer $\Omega$ par $\Omega_{1}$. Soit $u \in H\left(\Lambda_{\varepsilon G}, \tilde{r}^{2}\right),(P-z) u=v$ avec $z$ comme dans la Proposition 4.1. Par ellipticité, on montre d'abord à l'aide des arguments de [5], que:

$$
\|u\|_{H\left(A_{\varepsilon G}, C \Omega_{2}, \tilde{r}^{2}\right)} \leqq C\left(\|v\|_{H\left(A_{\varepsilon G}, 1\right)}+h^{2}\|u\|_{H\left(A_{\varepsilon G}, \tilde{r}^{2}\right)}\right) .
$$

D'autre part, en réalisant $Q$ par des contours singuliers comme avant dans cette section, on $a$ :

$$
(Q-z) U u=U v+r, \text { dans } \Omega_{1},
$$


où:

$$
\|r\|_{\Phi_{\varepsilon}, \Omega_{1}} \leqq C h^{3}\|u\|_{H\left(\Lambda_{\varepsilon}, \tilde{r}^{2}\right)} .
$$

Appliquant la Proposition 4.1, on trouve:

$$
\|U u\|_{\Phi_{\iota}, \Omega_{2}} \leqq C\left(h^{-1}\|v\|_{H\left(\Lambda_{\epsilon G}, 1\right)}+h^{2}\|u\|_{H\left(\Lambda_{\varepsilon G}, \hat{r}^{2}\right)}\right) .
$$

Sommant (4.19) et (4.22), on trouve à l'aide de (3.9):

$$
\|u\|_{H\left(A_{\varepsilon G}, \hat{r}^{2}\right)} \leqq C\left(h^{-1}\|v\|_{H\left(\Lambda_{\varepsilon}, 1\right)}+h^{2}\|u\|_{H\left(A_{\varepsilon G}, \tilde{r}^{2}\right)}\right) .
$$

Pour $h$ assez petit le dernier terme de (4.23) s'absorbe, et on a alors une inégalité à priori qui montre que $z$ ne peut pas être une résonance. Ceci achève la démonstration du Théorème 0.1 .

\section{Appendice}

\section{A.1. Variétés invariantes hyperboliques}

On rappelle la définition et quelques propriétés des variétés invariantes hyperboliques étudiées par exemple dans $[6,9]$.

On démontre ensuite quelques résultats sur la structure symplectique de ces variétés dans le cas d'un flot hamiltonien.

Soit $p \in C^{\infty}\left(T^{*} \mathbb{R}^{n}\right)$ un hamiltonien et $F^{t}$ le flot $\exp \left(t H_{p}\right)$, que l'on supposera complet pour simplifier la discussion. Si $A \in G L_{m}(\mathbb{R})$ on pose $m(A)$ $=\inf _{\|x\|=1}(\|A x\|)$ et on note $\|A\|$ la norme de $A$.

On choisit ensuite des normes sur $T_{\varrho}\left(T^{*} \mathbb{R}^{n}\right)$ qui dépendent continument de $\varrho$ pour $\varrho$ dans un borné assez grand de $T^{*} \mathbb{R}^{n}$. (La définition sera indépendante du choix des normes.)

Définition A.1. Soit $\Sigma$ une sous variété compacte de $T^{*}\left(\mathbb{R}^{n}\right)$ de classe $C^{1}$ et invariante par le flot $F^{t}$. On dit que $\Sigma$ est $r$-normalement hyperbolique pour $r \geqq 1$, si on a les propriétés suivantes:

i) $T_{\Sigma}\left(T^{*} \mathbb{R}^{n}\right)$ se décompose en somme directe de trois sous-fibrés $C^{0}: T_{\Sigma}\left(T^{*} \mathbb{R}^{n}\right)$ $=T \Sigma \oplus N_{+} \oplus N_{-}$, où $T \Sigma, N_{+}, N_{-}$, sont invariants par $d F^{t}$.

ii) on pose pour $\varrho \in \Sigma: V_{\varrho}^{t}=d F^{t}\left|T_{\varrho} \Sigma, N_{+1-, \varrho}^{t}=d F^{t}\right| N_{+1-, \varrho}$. Alors $\exists a>0, T>0$ tels que:

$$
\begin{gathered}
\inf _{\varrho \in \Sigma} m\left(N_{+, \varrho}^{t}\right) \geqq e^{a t}, \forall t \geqq T, \\
\sup _{\varrho \in \Sigma}\left\|N_{-, \varrho}^{t}\right\| \leqq e^{-a t}, \forall t \geqq T, \\
\inf _{\varrho \in \Sigma} m\left(N_{+, \varrho}^{t}\right) \geqq \sup _{\varrho \in \Sigma}\left\|V_{\varrho}^{t}\right\|^{r}, \forall t \geqq T, \\
\sup _{\varrho \in \Sigma}\left\|N_{-, \varrho}^{t}\right\| \leqq \inf _{\varrho \in \Sigma} m\left(V_{\varrho}^{t}\right)^{r}, \forall t \geqq T .
\end{gathered}
$$

On voit donc que si $\sum$ est $r$-normalement hyperbolique, $d F^{1}$ est exponentiellement expansif sur $N_{+}$, exponentiellement contractif sur $N_{-}$, dilate plus $N_{+}$que $T \Sigma$, et contracte plus $N$ - que $T \Sigma$.

Si $\Sigma$ est $r$-normalement hyperbolique, il existe d'après les résultats de [6] des variétés $C^{r} F^{t}$ invariantes $\Sigma_{ \pm}$tangentes sur $\Sigma$ à $T \Sigma \oplus N_{ \pm}$et fibrées au dessus de $\Sigma$ par des variétés $C^{r} \Gamma_{-, \varrho}$ pour $\varrho \in \Sigma$, tangentes en $\varrho$ à $N_{ \pm \varrho} . \Gamma_{ \pm, \varrho}$ sont caractérisées 
localement près de $\Sigma$ par:

$$
\begin{gathered}
\forall \varrho^{\prime} \in \Gamma_{+, \varrho} \text { avec } \operatorname{dist}\left(\varrho, \varrho^{\prime}\right) \leqq \varepsilon \text { on } a: \\
\operatorname{dist}\left(F^{-t}\left(\varrho^{\prime}\right), F^{-t}(\varrho)\right) \leqq C e^{-(a-o(\varepsilon)) t} \operatorname{dist}\left(\varrho^{\prime}, \varrho\right), \forall t \geqq 0 .
\end{gathered}
$$

On a une caractérisation analogue pour $\Gamma_{-, \varrho}$.

On montre maintenant que si $\Sigma$ est une variété symplectique $r$-normalement hyperbolique avec $r>1, \Sigma$ vérifie les hypothèses $(H)$.

Proposition A.2. Soit $\Sigma$ une variété invariante compacte $C^{1} r$-normalement hyperbolique pour $r>1$ et symplectique. Alors: $\Sigma$ vérifie les hypothèses $(H)$.

Démonstration. Il suffit de vérifier (H.1). Montrons par exemple que $\forall \varrho \in \Sigma$, on $a$ : $N_{+, \varrho} \subset T_{\varrho} \Sigma^{\sigma}$. Soient $u \in N_{+, \varrho}, v \in T_{\varrho} \Sigma$. On a $\sigma(u, v)=\sigma\left(d_{\varrho} F^{-t} u, d_{\varrho} F^{-t} v\right)$, où $\sigma$ désigne la forme symplectique. Comme $\Sigma$ est symplectique, on voit que $m\left(V_{\varrho}^{-t}\right)^{-1} \geqq\left\|V_{\varrho}^{-t}\right\|$, $\forall \varrho \in \Sigma$, pour un choix convenable de normes. En utilisant aussi la définition A.1 ii), on voit que $\sigma\left(d_{\varrho} F^{-t} u, d_{\varrho} F^{-t} v\right) \rightarrow 0$ quand $t \rightarrow+\infty$, ce qui démontre la proposition.

On veut maintenant caractériser les points de $\Sigma$ où $\Sigma$ est symplectique, si $\Sigma$ est une variété invariante compacte $r$-normalement hyperbolique avec $r>1$. On commence par rappeler quelques notions de dynamique topologique. (Voir [1].) On note encore $F^{t}$ le flot induit sur $\Sigma$.

Définition A.3. Un point $\varrho \in \Sigma$ est dit récurrent si $\forall U$ voisinage de $\varrho$ dans $\Sigma, \forall T>0$, $\exists t>T$ tel que $F^{t} \varrho \in U$.

Un point $\varrho \in \Sigma$ est dit non errant si $\forall U$ voisinage de $\varrho$ dans $\Sigma, \forall T>0, \exists t>T$ tel que $F^{t}(U) \cap U \neq \emptyset$.

Si $\varrho \in \Sigma$, on note $\Omega(\varrho)$ l'ensemble des points d'accumulation de l'orbite de $\varrho$ par $F^{t}$.

Les points fixes et les orbites fermées sont des points récurrents, et tout point récurrent est non errant. La dynamique de $F^{t}$ restreint à l'ensemble des points non errants contient en fait toute l'information intéressante sur la dynamique de $F^{t}$ sur $\sum$. Enfin il est intéressant de noter que la propriété de $r$-normale hyperbolicité peut être caractérisée en considérant uniquement l'adhérence de l'ensemble des points récurrents (voir Theorème 2.17 de [6].)

Notons $S=\left\{\varrho \in \Sigma \mid T_{\varrho} \Sigma\right.$ est symplectique $\}$. $S$ est évidemment un ouvert $F^{t}$ invariant. On a la proposition suivante:

\section{Proposition A.4.}

i) si $S=\Sigma$, tous les points sont non errants.

ii) si $\Omega(\varrho) \cap S \neq \emptyset, \varrho \in S$.

On suppose maintenant que $\operatorname{dim} N_{+}=\operatorname{dim} N_{-}$.

iii) si tous les points de $\sum$ sont non errants, $S$ est un ouvert dense de $\Sigma$.

iv) si @ est un point récurrent, @ appartient à $S$.

Démonstration. Si $\Sigma$ est symplectique, $F^{t}$ conserve la forme volume $\omega=(\sigma)^{n}$, où $\sigma$ désigne aussi la forme symplectique sur $\Sigma$. Il est alors bien connu que tous les points de $\Sigma$ sont non errants (voir [10]).

ii) est immédiat et laissé au lecteur.

Montrons maintenant iv): on va supposer tout d'abord que $\varrho \in \Sigma$ appartient à une orbite fermée $\gamma$. Le cas où $\varrho$ est un point fixe se traite de la même façon. On 
considère l'application de Poincaré linéarisée associée à $\gamma$ pour le flot $F^{t}$ dans $T^{*} \mathbb{R}^{n}$. Si $\mu_{1}, \ldots, \mu_{2 n-2}$ sont les valeurs propres de cette application rangées par ordre de module croissant et si $t_{0}$ est la période de $\gamma$, les valeurs propres de $d_{\rho} F^{t_{0}}$ sont $1,1, \mu_{1}, \ldots, \mu_{2 n-2}$ et on $a: N_{-, \varrho}$ (respectivement $T_{\varrho} \Sigma, N_{+. \varrho}$ ) est l'espace propre généralisé associé à $\left(\mu_{1}, \ldots, \mu_{k}\right)$ (respectivement $\left.\left(\mu_{k+1}, \ldots, \mu_{p-1}, 1\right),\left(\mu_{p}, \ldots, \mu_{2 n-2}\right)\right)$. La condition de $r$-normale hyperbolicité s'écrit, en remplacant éventuellement $t_{0}$ par $m t_{0}$ pour $m \in \mathbb{N}$ :

$$
\left|\mu_{p}\right| \geqq\left(\sup \left\{\left|\mu_{p-1}\right|, 1\right\}\right)^{r},\left|\mu_{k}\right| \leqq\left(\inf \left\{\left|\mu_{k+1}\right|, 1\right\}\right)^{r},
$$

et on $a:\left|\mu_{p}\right|>1,\left|\mu_{k}\right|<1$.

Ceci entraine que $T_{e} \Sigma$ est $\sigma$-orthogonal à $N_{-, \varrho} \oplus N_{+, \varrho}$ comme $\operatorname{dim} N_{+, \varrho}$ $=\operatorname{dim} N_{-, \varrho}$, donc $T_{\varrho} \Sigma$ est symplectique. Soit maintenant $\varrho \in \Sigma$ un point récurrent. Soit $U(\varepsilon)$ un voisinage de taille $\varepsilon$ de $\varrho$ dans $\Sigma$ et $T_{0} \gg 1$, tel que $F^{T_{0}}(\varrho) \in U(\varepsilon)$. Si $\varrho^{\prime}=F^{T_{0}}(\varrho), d_{\varrho} F^{T_{0}}$ envoie $N_{-, \varrho} \oplus T_{\varrho} \Sigma \oplus N_{+, \varrho}$ sur les fibrés correspondants en $\varrho^{\prime}$, et les fibrés en $\varrho^{\prime}$ sont proches des fibrés en $\varrho$ dans la topologie grassmanienne car $\varrho^{\prime} \in U(\varepsilon)$.

C'est un exercice facile sur les formes normales dans un espace symplectique de vérifier qu'il existe une application symplectique $R_{\varepsilon}=\mathrm{Id}+o(\varepsilon)$, qui envoie $N_{+/-, Q^{\prime}}$ et $T_{Q^{\prime}} \Sigma$ sur $N_{+/-, Q}$ et $T_{Q} \Sigma$, qui sont alors des sommes d'espaces propres pour $R_{\varepsilon} d_{\varrho} F^{T_{0}}=T_{\varepsilon}$, et si $T_{0}$ est assez grand, $\varepsilon$ assez petit, on $a$ :

$$
\begin{gathered}
m\left(T_{\varepsilon} \mid N_{+, \varrho}\right) \geqq\left\|T_{\varepsilon} \mid T_{\varrho} \Sigma\right\| r^{\prime} \text {, avec } 1<r^{\prime}<r . \\
\left\|T_{\varepsilon} \mid N_{-, \varrho}\right\| \leqq m\left(T_{\varepsilon} \mid T_{\varrho} \Sigma\right)^{r^{\prime}}, \text { et } m\left(T_{\varepsilon} \mid N_{+, \varrho}\right)>1,\left\|T_{\varepsilon} \mid N_{-, \varrho}\right\|<1 .
\end{gathered}
$$

On en déduit comme dans le cas de la trajectoire fermée que $T_{Q} \Sigma$ est symplectique.

Montrons maintenant iii). Si $\varrho \in \Sigma$ est un point non errant, dans tout voisinage de $\varrho$, il part une trajectoire de $H_{p}$ qui $y$ revient après un temps arbitrairement long. L'argument utilisé plus haut montre que le point de départ de cette trajectoire appartient à $S$, ce qui montre que $S$ est dense dans $\Sigma$.

Pour finir on donne des exemples de variétés invariantes compactes $r$-normalement hyperboliques et symplectiques.

Proposition A.5. Soient $0<\varepsilon_{1}<\varepsilon_{0}$ petits. Soit $p_{0} \in C^{\infty}\left(T^{*} \mathbb{R}^{n}\right)$ un hamiltonien tel que l'ensemble des trajectoires captées $K_{0}$ vérifie: $K_{0} \cap p_{0}^{-1}\left(\left[-\varepsilon_{0}, \varepsilon_{0}\right]\right)=\Sigma_{0}$ $\cap p_{0}^{-1}\left(\left[-\varepsilon_{0}, \varepsilon_{0}\right]\right)$, où $\Sigma_{0}$ est une variété $C^{1} r$-normalement hyperbolique pour $r>1$ et symplectique. Alors si $p=p_{0}+\delta p$ avec $\delta p$ assez petit dans $C^{\infty}\left(T^{*} \mathbb{R}^{n}\right)$ et si $\Omega$ est un petit voisinage de $K_{0} \cap p_{0}^{-1}\left(\left[-\varepsilon_{0}, \varepsilon_{0}\right]\right)$, l'ensemble des trajectoires captées $K$ associé à $p$ vérifie: $K \cap p^{-1}\left(\left[-\varepsilon_{1}, \varepsilon_{1}\right]\right) \cap \Omega=\Sigma \cap p^{-1}\left(\left[-\varepsilon_{1}, \varepsilon_{1}\right]\right) \cap \Omega$, où $\Sigma$ est une variété $C^{1}$ $r$-normalement hyperbolique et symplectique.

Démonstration. Il suffit d'appliquer le Thèoreme 4.1 de [6].

Exemple A.6. On considère:

$$
p_{0}(x, \xi)=\sum_{1 \leqq j \leqq n-d} \lambda_{j}\left(x_{j}^{2}+\xi_{j}^{2}\right) / 2+\sum_{n-d+1 \leqq j \leqq n} \lambda_{j}\left(\xi_{j}^{2}-x_{j}^{2}\right) / 2 .
$$

$p_{0}$ provient modulo des changements linéaires de coordonnées de $\xi^{2}+V(x)$, symbole principal de l'opérateur de Schrödinger semi-classique $P=-h^{2} \Delta$ $+V(x)$ avec

$$
V=\sum_{1 \leqq j \leqq n-d} \mu_{j} x_{j}^{2}-\sum_{n-d+1 \leqq j \leqq n} \mu_{j} x_{j}^{2} \text {, et } \mu_{j}=\lambda_{j}^{2} / 4 .
$$


Si $x=\left(x_{1}, \ldots, x_{n-d}\right), x^{\prime \prime}=\left(x_{n-d+1}, \ldots, x_{n}\right)$, alors pour $0<\varepsilon_{1}<\varepsilon_{2}, \sum=p_{0}^{-1}\left(\left[\varepsilon_{1}, \varepsilon_{2}\right]\right)$ $\cap\left\{x^{\prime \prime}=\xi^{\prime \prime}=0\right\}$ est une variété $r$-normalement hyperbolique avec $r>1$. D'après la Proposition A.5 on obtient d'autres exemples en perturbant un peu $p_{0}$.

\section{A.II. Construction d'une fonction fuite}

On se place sous les hypothèses $(H)$ et on veut montrer l'existence d'une fonction $G_{0}$ de classe $C^{2}$, s'annulant à l'ordre $2 \operatorname{sur} \Sigma$, telle le hessien transversal de $G_{0}$ en tout point $\varrho$ de $\Sigma$ soit donné par:

$$
G_{0}^{\prime \prime}\left(v_{+}+v_{-}\right)=\left\|v_{+}\right\|^{2}-\left\|v_{-}\right\|^{2}, v_{ \pm} \in N_{ \pm, \varrho} .
$$

Il suffit de construire $G_{0}$ localement près d'un point $\varrho_{0}$ de $\Sigma$. Soient $g_{1}, \ldots, g_{2 d}$ des fonctions de classe $C^{1}$ à différentielles indépendantes en $\varrho_{0}$ telles que $\Sigma$ est donnée près de $\varrho_{0}$ par les équations $g_{1}=\ldots=g_{2 d}=0$. Pour simplifier, on va supposer que $\Sigma$ est une sous-varieté de $\mathbb{R}^{n}$. On veut alors trouver une fonction $G_{0}$ de classe $C^{2}$ telle que:

$$
G_{0}(x)=\Sigma \Sigma a_{i, j}(x) g_{i}(x) g_{j}(x)+o\left(d(x, \Sigma)^{2}\right),
$$

où les $a_{i, j}$ sont des fonctions de classe $C^{0}$ déterminées par (A.2). On va construire $G_{0}$ en régularisant $a_{i, j}$ et $g_{i}$.

On commence par montrer un lemme:

Lemme A.6. Il existe une fonction $c(x), C^{\infty}$ hors de $\Sigma$ telle que $\nabla c=O(1)$, $\nabla^{2} c=O\left(c^{-1}\right)$ et $c \sim d(\cdot, \Sigma)$ près de $\varrho_{0}$.

Démonstration. Soit $V$ un voisinage de $\Sigma$. On prend $\varepsilon>0$ assez petit et un ensemble de points $x_{i}, i \in \mathbb{N}$, tel que les boules $B\left(x_{i}, \varepsilon d\left(x_{i}, \Sigma\right)\right)$ sont disjointes et pour tout $x \in V$ il existe $i$ tel que $B(x, \varepsilon d(x, \Sigma)) \cap B\left(x_{i}, \varepsilon d\left(x_{i}, \Sigma\right)\right)$ n'est pas vide. Alors les $B\left(x_{i}, 4 \varepsilon d\left(x_{i}, \Sigma\right)\right)$ forment un recouvrement localement fini. Soit $\chi \in C_{0}^{\infty}(B(0,1))$ une fonction troncature avec $\chi=1 \operatorname{sur} B(0,1 / 2)$. On pose alors

$$
f(x)=\sum_{i \in \mathbb{N}} d\left(x_{i}\right)^{2} \chi\left(\left(x-x_{i}\right) / 8 \varepsilon d\left(x_{i}\right)\right) .
$$

Il est facile de vérifier que $c(x)=(f(x))^{1 / 2}$ possède les propriétés du lemme.

On pose maintenant $a^{*}(x)=\int \chi(y) a(x-c(x) y) d y, g^{*}(x)=\int \chi(y) g(x-c(x) y) d y$, où $\chi \in C_{0}^{\infty}\left(\mathbb{R}^{2 n}\right)$ est une fonction radiale telle que,

$$
\int \chi(x) d x=1
$$

Ici a est un des $a_{i, j}$ et $g$ un des $g_{j}$. On a la proposition suivante:

Proposition A.7. On a quand $d(\cdot, \Sigma) \rightarrow 0$ :

(i) $a-a^{*}=o(1), g-g^{*}=o(c)$,

(ii) $\nabla_{x} a^{*}=o(1 / c), \nabla_{x} g^{*}=O(1)$,

(iii) $\nabla_{x}^{2} a^{*}=o\left(1 / c^{2}\right), \nabla_{x}^{2} g^{*}=O(1 / c)$.

Démonstration. On va montrer uniquement la proposition pour $a^{*}$, le cas de $g^{*}$ se traitant de la même façon.

(i): On a $a(x)-a^{*}(x)=\int \chi(y)(a(x)-a(x-c(x) y)) d y=o(1)$.

(ii): Par changement de variables on $a: a^{*}(x)=c^{-n} \int \chi((x-y) / c) a(y) d y$. On 
obtient que $\partial_{i} a^{*}(x)=A_{0}+\sum_{1 \leqq j \leqq n} A_{j}$, avec: $A_{0}=c^{-n-1} \int\left(\partial_{i} \chi\right)((x-y) / c) a(y) d y$, $A_{j}=c^{-n-1}\left(\partial_{j} c\right) \int f_{j}((x-y) / c) a(y) d y, f_{i}=y_{i} \partial_{i} \chi+\chi$. Par un nouveau changement de variables, on $a$ : $A_{0}=c^{-1} \int\left(\partial_{i} \chi(y)\right) a(x-c y) d y=o(1 / c)$, grâce à (A.3), et $A_{j}=\left(\partial_{i} c / c\right) \int f_{i}(y) a(x-c y) d y=o(1 / c)$, car $\int f(y) d y=0$. On a donc démontré (ii).

(iii): Un calcul facile donne:

$$
\begin{aligned}
\partial_{k} A_{0}= & -c^{-n-2}\left(\partial_{k} c\right) \sum_{1 \leqq j \leqq n} \int f_{i, j}((x-y) / c) a(y) d y \\
& -c^{-n-2}\left(\partial_{k} c\right) \int\left(\partial_{i} \chi\right)((x-y) / c) a(y) d y+c^{-n-2} \int\left(\partial_{i, k}^{2} \chi((x-y) / c)\right) a(y) d y,
\end{aligned}
$$

avec $f_{i, j}=y_{j}\left(\partial_{i, j}^{2} \chi\right)+\partial_{i} \chi$. Par le même argument que plus haut on obtient que: $A_{0}=o\left(1 / c^{2}\right)$, car $\int f_{i, j}(y) d y=\int \partial_{i, k}^{2} \chi(y) d y=\int \partial_{i} \chi(y) d y=0$. De même on a:

$$
\begin{aligned}
\partial_{k} A_{1}= & c^{-n-2}\left(\partial_{i, k}^{2} c\right) \int f_{1}((x-y) / c) a(y) d y-c^{-n-2}\left(\partial_{i} c\right)\left(\partial_{k} c\right) \int f_{1}((x-y) / c) a(y) d y \\
& \left.-c^{-n-2}\left(\partial_{i} c\right)\left(\partial_{k} c\right) \sum_{1 \leqq j \leqq n} \int\left(y_{j} \partial_{j} f_{1}+f_{1}\right)(x-y) / c\right) a(y) d y \\
& +c^{-n-2} \partial_{i} c \int \partial_{k} f_{1}((x-y) / c) a(y) d y .
\end{aligned}
$$

On a encore $\partial_{k} A_{1}=o\left(1 / c^{2}\right)$, car $\int \partial_{k} f_{1}(y) d y=\int\left(y_{j} \partial_{j} f_{1}+f_{1}\right) d y=0$.

On pose alors $G_{0}=\sum \sum a_{i, j}^{*} g_{i}^{*} g_{j}^{*}$. Grace à (i) on a $G_{0}-\sum \sum a_{i, j} g_{i} g_{j}=o\left(c^{2}\right)$. Il reste à montrer que $G_{0}$ est $C^{2}$. Grace à (ii), on voit que $\nabla_{x} G_{0}=O(c)$ et grace à (iii) que $\nabla_{x}^{2} G_{0}$ a une limite quand $c \rightarrow 0$.

\section{Bibliographie}

(D’autres références sur les résonances sont indiquées dans [5], et on se limite ici aux travaux qui jouent un rôle pour les techniques utilisées.)

1. Abraham, R., Marsden, J.: Foundations of mechanics. New York: Benjamin Cumming 1978

2. Abraham, R., Robbin, J.: Transversal mappings and flows. New York: Cummings 1967

3. Fenichel, N.: Persistence and smoothness of invariant manifolds for flows. Indiana Univ. Math. J. 21 (3), 193-226 (1971)

4. Gérard, C., Sjöstrand, J.: Semiclassical resonances generated by a closed trajectory of hyperbolic type. Commun. Math. Phys. 108, 391-421 (1987)

5. Helffer, B., Sjöstrand, J.: Résonances en limite semiclassique. Bull. S.M.F., Mémoire Vol. 24/25 (1986)

6. Hirsch, W.M., Pugh, C.C., Shub, M.: Invariant manifolds. Lecture Notes in Mathematics, Vol. 583. Berlin, Heidelberg, New York: Springer 1977

7. Oseledets, V.I.: A multiplicative ergodic theorem. Lyapunov characteristic exponents for dynamical systems. Trans. Moscow Math. Soc. 19, 197-231 (1968)

8. Sjöstrand, J.: Singularités analytiques microlocales. Astérisque, Vol. 95 (1982)

9. Sjöstrand, J.: Semiclassical resonances generated by non-degenerate critical points. Lecture Notes in Mathematics. Berlin, Heidelberg, New York: Springer 1987

10. Smale, S.: Differentiable dynamical systems. Bull. A.M.S., Vol. 73, 747-817 (1967)

11. Briet, Ph., Combes, J.M., Duclos, P.: On the location of resonances for Schrödinger operators in the semiclassical limit. II. Commun. PDE 12 (2), 201-222 (1987)

Communicated by B. Simon

Received June 5, 1987; in revised form October 13, 1987 
